\title{
DYNAMICS OF TWO LINEARLY ELASTIC BODIES CONNECTED BY A HEAVY THIN SOFT VISCOELASTIC LAYER
}

\author{
ELENA BONETTI, GIOVANNA BONFANTI, CHRISTIAN LICHT, AND RICCARDA ROSSI
}

\begin{abstract}
In this paper we extend the asymptotic analysis in 18, performed on a structure consisting of two linearly elastic bodies connected by a thin soft nonlinear KelvinVoigt viscoelastic adhesive layer, to the case in which the total mass of the layer remains strictly positive as its thickness tends to zero.

We obtain convergence results by means of a nonlinear version of Trotter's theory of approximation of semigroups acting on variable Hilbert spaces. Differently from the limit models derived in [18, in the present analysis the dynamic effects on the surface on which the layer shrinks do not disappear. Thus, the limiting behavior of the remaining bodies is described not only in terms of their displacements on the contact surface, but also by an additional variable that keeps track of the dynamics in the adhesive layer.
\end{abstract}

\section{INTRODUCTION}

PDE systems coupling bulk and surface equations play an important role in several applications. In particular, they are used to describe different physical situations in which two spatial scales are involved: a macroscopic scale for the bulk domain and a microscopic scale to capture dynamics on a thin layer located at the boundary. Among others, models for contact with adhesion between rigid bodies represent an important application of this kind of approach. Indeed, these models couple mechanical and thermal properties of the involved bodies and of the microscopic configurations of the thin adhesive layer between the bodies.

In a macroscopic description, this layer is considered as a part of the boundaries and dynamics of the physical variables are described by boundary equations. This feature occurs, e.g., in the models for contact with adhesion between a viscoelastic body and a rigid support analyzed in, e.g., [3], 4], and [5]. Such models are derived from the theory for damage in thermoelastic materials by FrÉmond [10, 11. Specifically, the related energy functionals and dissipation potentials are written both in the bulk and on the surface and, accordingly, bulk and surface equations are recovered via a generalization of the principle of virtual powers. The main idea is to account for the effects of the microscopic forces, responsible for the degradation of the adhesion on the interface between body and support, in the energy balance. While the PDE systems from [3, [4, and [5] are rate-dependent, delamination can also be treated as a rate-independent phenomenon, see e.g. [15, 21. In that modeling context as well, the microscopic damage in the interface is assumed to influence the strength of the adhesion and unilateral conditions are accounted for to ensure non-penetrability between the adhering bodies.

A possible validation of this kind of models, coupling bulk and surface phenomena, could be provided by deriving the surface equations from equations set on a thin layer, as the thickness of the layer tends to zero. This kind of asymptotic analysis has been tackled in the literature using different analytical techniques and modeling approaches. One possibility is to develop a formal asymptotic expansion method as in 114, 17, 12, For damage and delamination, we refer to the asymptotic analyses carried out in [1, 2. In the context of rateindependent modeling of delamination, instead, $\Gamma$-convergence type techniques were used in 19 to show that Energetic solutions to a system for isotropic damage converge to an Energetic solution of a delamination model

This work was partially carried out during a visit of C.L. at the Sezione di Matematica of DICATAM (University of Brescia), also supported by Gruppo Nazionale per l'Analisi Matematica, la Probabilità e le loro Applicazioni (GNAMPA) of the Istituto Nazionale di Alta Matematica (INdAM) . 
as the thickness of the layer between the two bulk bodies, where damage occurs, tends to zero. Indeed, the Energetic weak solvability notion for rate-independent processes, consisting of an energy-dissipation balance and of a stability condition that involves the minimization of a suitable functional, allows for the usage of the variational techniques at the core of the analysis in [19] (see also [8, 9]).

A rigorous approach based on variational convergences techniques has been carried out for this kind of problems, in the rate-dependent framework, in a series of papers, cf. e.g. [16, 17, 18, 13. In particular, this paper follows up on the analysis developed in [18, where the authors derived an asymptotic model for the dynamics of two linearly elastic bodies connected by a thin viscoelastic layer by means by of a nonlinear version of Trotter's theory of approximation of semigroups, cf. [22]. More specifically, in [18] the model was obtained by studying the asymptotic behavior, as some parameters accounting for geometrical and mechanical data vanished, of the structure consisting of the two adhering bodies, perfectly bonded through the adhesive layer. The analysis was carried out under the further assumption that the total mass of the adhesive layer was vanishing. Hence, the limit model obtained in [18] describes for the dynamics of two adhering bodies subject to a mechanical constraint along the surface $S$ the layer shrinks to. Its constitutive equation is of the same type as that for the layer (nonlinear viscoelastic of Kelvin-Voigt type).

In this paper, we aim to extend the asymptotic analysis in [18, by considering the case in which the total mass remains strictly positive; indeed, this is what the term 'heavy' in the title refers to. As in [18, the cornerstone of our analysis will be the reformulation of the original problem, in which the interface is given with a positive thickness, in terms of a nonlinear evolution equation in a Hilbert space of admissible states with finite mechanical energy, governed by a suitable maximal monotone operator. Our convergence result shall then be obtained by resorting to a nonlinear version of Trotter's theory of approximation of semigroups of operators acting on variable Hilbert spaces, see [20]. Albeit relying on the same theoretical tools as those of [18], our analysis here is significantly different. Indeed, since the dynamic effects in the thin layer do not disappear, the limiting contact condition between the two remaining bodies shall not only involve their displacements along the interface but an additional variable, too, which accounts for the asymptotic behavior of the layer and whose analytical treatment within Trotter's theory calls for suitable arguments. Of course, such a variable may be eliminated so that the constraint appears as viscoelastic with long memory, cf. Sec. 5 .

Plan of the paper. In Section 2 we specify the setting of the problem, starting from the formulation of the model when the thickness of the interface is positive. Then, in Section 3 we recast this problem as an abstract evolution equation in a Hilbert space, governed by a suitable maximal monotone operator. Staying with this formulation, in Section 4 we carry out our asymptotic analysis, as the thickness of the layer between the two adhering bodies vanishes, by means of Trotter's theory. In this way we prove the main result of this paper, Theorem 4.15. Finally, in Section 5 we give some further comments on our result, and hint at some extensions.

Throughout this paper, we will use the following notation.

Notation 1.1 (General notation). We will denote the orthonormal basis of $\mathbb{R}^{3}$ by $\left(e_{1}, e_{2}, e_{3}\right)$. Given a vector $\xi=\left(\xi_{1}, \xi_{2}, \xi_{3}\right) \in \mathbb{R}^{3}$, we will use the symbol $\widehat{\xi}$ for $\left(\xi_{1}, \xi_{2}\right)$, so that we will often write $\left(\widehat{\xi}, \xi_{3}\right)$ in place of $\left(\xi_{1}, \xi_{2}, \xi_{3}\right)$. The symbol $\operatorname{tr}(A)$ will denote the trace of a $\mathbb{R}^{3 \times 3}$ matrix, $\mathbb{R}_{\text {sym }}^{3 \times 3}$ the space of $(3 \times 3)$-symmetric matrices, equipped with the standard inner product, and $\operatorname{Lin}\left(\mathbb{R}_{\mathrm{sym}}^{3 \times 3}\right)$ the space of linear mappings from $\mathbb{R}_{\mathrm{sym}}^{3 \times 3}$ to $\mathbb{R}_{\mathrm{sym}}^{3 \times 3}$. Given two vectors $\xi, \zeta \in \mathbb{R}^{3}$, we shall denote by $\xi \otimes_{\mathrm{S}} \zeta$ their symmetrized tensor product, defined by

$$
\xi \otimes_{\mathrm{S}} \zeta \text { is the symmetric }(3 \times 3) \text {-matrix with entries } \frac{1}{2}\left(\xi_{i} \zeta_{j}+\xi_{j} \zeta_{i}\right) i, j=1, \ldots, 3 \text {. }
$$

With any subset $O \subset \mathbb{R}^{3}$ we will associate its characteristic function $\chi_{O}$, defined by $\chi_{O}(x)=1$ if $x \in O$, and $\chi_{O}(x)=0$ if $x \in \mathbb{R}^{3} \backslash O$.

Finally, throughout the paper, the symbol $C$ will denote various constants that may differ from one line to the other. 


\section{Setup of the PROBlem}

Let us specify the setup of our problem, namely the study of the dynamic response of a structure made up of two adhering bodies connected by a thin adhesive layer, subject to a given load. First of all, the reference configuration of the structure is a bounded connected open subset $\Omega \subset \mathbb{R}^{3}$ with a Lipschitz boundary $\partial \Omega$. Hereafter, we will denote by $S$ the set

$$
S:=\Omega \cap\left\{\left(x_{1}, x_{2}, x_{3}\right) \in \mathbb{R}^{3}: x_{3}=0\right\} \text { and assume that its Hausdorff measure } \mathcal{H}^{2}(S)>0 .
$$

In what follows, we shall identify $S$ with its projection onto $\mathbb{R}^{2}$ and therefore treat it as a subset of $\mathbb{R}^{2}$.

Notation 2.1. For a function $u \in H^{1}\left(\Omega \backslash S ; \mathbb{R}^{3}\right)$, we will denote by $u^{+}\left(u^{-}\right.$, respectively), its restriction to the open set $\Omega^{ \pm}:=\left\{x=\left(x_{1}, x_{2}, x_{3}\right) \in \Omega: \pm x_{3}>0\right\}$, which is a function in $H^{1}\left(\Omega^{ \pm}\right)$. The symbols $\gamma_{S}\left(u^{+}\right)$and $\gamma_{S}\left(u^{-}\right)$will denote the traces of $u^{+}$and $u^{-}$, respectively, on the set $S$. Moreover, we will use the notation

$$
\text { jump of } u \text { across } S: \quad \llbracket u \rrbracket:=\gamma_{S}\left(u^{+}\right)-\gamma_{S}\left(u^{-}\right) \text {. }
$$

Throughout the paper, we will assume that there exists $\varepsilon_{0}>0$ such that

$$
B_{\varepsilon_{0}}:=\left\{x=\left(x_{1}, x_{2}, x_{3}\right) \in \Omega:\left|x_{3}\right|<\varepsilon_{0}\right\} \text { is equal to } S \times\left(-\varepsilon_{0}, \varepsilon_{0}\right) .
$$

For $0<\varepsilon<\varepsilon_{0}$, we will assume that the adhesive occupies the layer $B_{\varepsilon}:=S \times(-\varepsilon, \varepsilon)$, while the two adhering bodies shall occupy the sets $\Omega_{\varepsilon}^{ \pm}:=\left\{x=\left(x_{1}, x_{2}, x_{3}\right) \in \Omega: \pm x_{3}>\varepsilon\right\}$. We let $\Omega_{\varepsilon}:=\Omega_{\varepsilon}^{+} \cup \Omega_{\varepsilon}^{-}=\Omega \backslash \bar{B}_{\varepsilon}$. We will use the notation

$$
S_{\varepsilon}^{ \pm}:=\left\{x \in \Omega: x_{3}= \pm \varepsilon\right\}, \quad B_{\varepsilon}^{+}:=S \times(0, \varepsilon), \quad B_{\varepsilon}^{-}:=S \times(-\varepsilon, 0)
$$

and assume that adhesive and adhering bodies are perfectly stuck together along $S_{\varepsilon}:=S_{\varepsilon}^{+} \cup S_{\varepsilon}^{-}$. This means that the jumps across $S_{\varepsilon}$ both of the displacement $u$ and of the normal stress $\sigma e_{3}$ are zero, cf. (2.8d) and (2.8e) below.

We consider a partition of $\partial \Omega=\Gamma_{\mathrm{D}} \cup \Gamma_{\mathrm{N}}$ such that $\Gamma_{\mathrm{D}}$ has positive two-dimensional Hausdorff measure and positive distance from $B_{\varepsilon_{0}}$; we assume that, during the time interval $(0, T)$, the structure is clamped on $\Gamma_{\mathrm{D}}$ and subjected to volumetric and surface forces (on $\Gamma_{\mathrm{N}}:=\partial \Omega \backslash \Gamma_{\mathrm{D}}$ ), with densities $f$ and $g$, respectively. We let $\Gamma_{\mathrm{D}}^{ \pm}:=\Gamma_{\mathrm{D}} \cap\left\{ \pm x_{3}>0\right\}$.

The adhering bodies are modeled as linearly elastic materials with a strain energy density $W$ such that

$$
W(x, e)=\frac{1}{2} a(x) e \cdot e \quad \text { for a.a. } x \in \Omega \text { and for all } e \in \mathbb{R}_{\mathrm{sym}}^{3 \times 3}, \quad \text { with }
$$

$a \in L^{\infty}\left(\Omega ; \operatorname{Lin}\left(\mathbb{R}_{\mathrm{sym}}^{3 \times 3}\right)\right)$ such that $\exists \alpha, \beta>0$ for a.a. $x \in \Omega$ for all $e \in \mathbb{R}_{\mathrm{sym}}^{3 \times 3}: \quad \alpha|e|^{2} \leq a(x) e \cdot e \leq \beta|e|^{2}$.

The adhesive is assumed homogeneous, isotropic, and 'viscoelastic of Kelvin-Voigt generalized type'. Its strain energy density reads as

$$
W_{\lambda, \mu}(e):=\frac{\lambda}{2}|\operatorname{tr}(e)|^{2}+\mu|e|^{2} \quad \text { for all } e \in \mathbb{R}_{\mathrm{sym}}^{3 \times 3}, \quad \text { with } \lambda, \mu>0 \text { the Lamé constants. }
$$

We will denote by $\mathrm{D} W_{\lambda, \mu}(e)$ its differential at any $e \in \mathbb{R}_{\mathrm{sym}}^{3 \times 3}$. Observe that

$$
2 W_{\lambda, \mu}(e)=\mathrm{D} W_{\lambda \mu}(e) \cdot e \geq 2 \mu|e|^{2} \quad \text { for all } e \in \mathbb{R}_{\mathrm{sym}}^{3 \times 3} .
$$

Dissipation in the adhesive is modeled through a dissipation potential $\mathcal{D}: \mathbb{R}_{\mathrm{sym}}^{3 \times 3} \rightarrow[0, \infty)$ such that $\mathcal{D}$ is convex and fulfils

$$
\exists p \in[1,2], \quad \exists \alpha^{\prime}, \beta^{\prime}>0 \quad \forall e \in \mathbb{R}_{\mathrm{sym}}^{3 \times 3}: \quad \alpha^{\prime}|e|^{p} \leq \mathcal{D}(e) \leq \beta^{\prime}\left(|e|^{p}+1\right) ;
$$

we will denote by $\partial \mathcal{D}: \mathbb{R}_{\text {sym }}^{3 \times 3} \rightrightarrows \mathbb{R}_{\text {sym }}^{3 \times 3}$ its subdifferential in the sense of convex analysis. Indeed, in system (2.8) below the functional $\mathcal{D}$ shall be multiplied by a positive constant $b$ that accounts for the intensity of viscous 
effects. Finally, we assume that the density $\gamma$ of the structure takes two different positive values in $\Omega_{\varepsilon}$ and $B_{\varepsilon}$, namely

$$
\begin{aligned}
& \gamma(x)=\left\{\begin{array}{ll}
\rho^{*}(x) & \text { for a.e. } x \in \Omega_{\varepsilon}, \\
\rho & \text { for a.e. } x \in B_{\varepsilon},
\end{array}\right. \text { with } \\
& \rho^{*}: \Omega \rightarrow(0, \infty) \text { a measurable function s.t. } \exists 0<\bar{\rho}_{m}<\bar{\rho}_{M} \text { for a.a. } x \in \Omega: \quad \bar{\rho}_{m} \leq \rho^{*}(x) \leq \bar{\rho}_{M} .
\end{aligned}
$$

Then, the model for the dynamic response of the structure, in the case the thin adhesive layer still has a 'positive thickness', is described by the following PDE system.

$$
\begin{aligned}
& \gamma u_{t t}-\operatorname{div}(\sigma)=f \\
& \sigma=a(x) e(u) \\
& \sigma=\lambda \operatorname{tr}(e(u)) \mathbb{I}+2 \mu e(u)+b \xi \\
& \llbracket \sigma e_{3} \rrbracket=0 \\
& \llbracket u \rrbracket=0 \\
& u=0 \\
& \sigma n=g
\end{aligned}
$$$$
\text { in } \Omega \times(0, T) \text {, }
$$

in $\Omega_{\varepsilon} \times(0, T)$,

in $B_{\varepsilon} \times(0, T)$,

with $\xi \in \partial \mathcal{D}\left(e\left(u_{t}\right)\right)$,

on $S_{\varepsilon}^{ \pm} \times(0, T)$,

on $S_{\varepsilon}^{ \pm} \times(0, T)$,

on $\Gamma_{\mathrm{D}} \times(0, T)$,

on $\Gamma_{\mathrm{N}} \times(0, T)$,

where $\mathbb{I}$ denotes the identity matrix and $e(u)$ the symmetric linearized strain tensor related to the displacement vector $u$, defined by $e_{i j}(u)=\frac{1}{2}\left(\partial_{j} u_{i}+\partial_{i} u_{j}\right), i, j=1, \ldots, 3$. We will supplement system (2.8) with the initial conditions

$$
u(0)=u_{0}, \quad u_{t}(0)=v_{0} \quad \text { in } \Omega .
$$

Note that the strong formulation (2.8) of the problem in the case the thickness of the adhesive layer is strictly positive indeed corresponds to the formulation of the momentum balance equations written in the two bulk domains. However, in what follows we will be able to provide an asymptotic result only for a variational (weak) formulation of system (2.8) with the Cauchy conditions (2.9), namely

Problem (P): Find $u: \Omega \times[0, T] \rightarrow \mathbb{R}^{3}$ sufficiently smooth fulfilling (2.8f), (2.9), and such that there exists $\xi \in \partial \mathcal{D}\left(e\left(u_{t}\right)\right)$ satisfying

$$
\begin{aligned}
& \int_{\Omega} \gamma u_{t t} \cdot v \mathrm{~d} x+\int_{\Omega_{\varepsilon}} a e(u) \cdot e(v) \mathrm{d} x+\int_{B_{\varepsilon}} \mathrm{D} W_{\lambda \mu}(e(u)) \cdot e(v) \mathrm{d} x+b \int_{B_{\varepsilon}} \xi \cdot e(v) \mathrm{d} x \\
& =\int_{\Omega} f \cdot v \mathrm{~d} x+\int_{\Gamma_{\mathrm{N}}} g \cdot v \mathrm{~d} \mathcal{H}^{2}(x)
\end{aligned}
$$

for all $v$ sufficiently smooth in $\Omega$ and vanishing on $\Gamma_{\mathrm{D}}$.

In the next section, we will show that Problem $(P)$ has a unique solution in a suitable sense. In Sec. 4 we will then determine the asymptotic behavior of the solutions to Problem $(\mathrm{P})$ when the quintuple of geometrical and mechanical data $(\varepsilon, \lambda, \mu, b, \rho)$ that characterize the structure is regarded as a quintuple of positive parameters $q_{n}:=\left(\varepsilon_{n}, \lambda_{n}, \mu_{n}, b_{n}, \rho_{n}\right)$, suitably converging to a limit $q_{\infty}$ (cf. the upcoming Hyp. 4.1).

\section{Existence and uniqueness of solutions to Problem $(P)$}

We will rigorously prove our existence result for Problem $(P)$ relying on the, by now classical, results from [6]. For the asymptotic analysis we shall resort to a nonlinear version of Trotter's theory of approximation of semigroups, acting on variable Hilbert spaces. This approach in fact relies on a reformulation of system (2.10) as an abstract evolutionary equation involving semigroups on suitable Hilbert spaces. In what follows, we recapitulate this formulation, as proposed in [18, and recall the existence result proved therein, cf. Theorem 3.2 ahead. Since we will keep the quintuple of parameters $q=(\varepsilon, \lambda, \mu, b, \rho)$ fixed in this section, in the following lines we shall not highlight the dependence of the solution $u$, and of the functionals/operators/spaces entering into the variational formulation of Problem $(P)$, on $q$. 
From now on we will assume that the body and the surface forces fulfill

$$
f \in \mathrm{BV}\left([0, T] ; L^{2}\left(\Omega ; \mathbb{R}^{3}\right)\right), \quad g \in W^{2, \infty}\left(0, T ; L^{2}\left(\Gamma_{\mathrm{N}} ; \mathbb{R}^{3}\right)\right) .
$$

Here and in what follows, $\mathrm{BV}([0, T] ; X)$ shall denote the subspace of $L^{1}(0, T ; X)$ consisting of all the elements whose distributional derivative with respect to time is a bounded Radon measure on $(0, T)$, valued in $X$. Along the footsteps of [18] we seek a solution to Problem $(P)$ of the form

$$
u=u^{\mathrm{e}}+u^{\mathrm{r}} .
$$

In (3.2), $u^{\mathrm{e}}$ is the unique solution of the 'stationary' problem

$$
u^{\mathrm{e}}(t) \in H_{\Gamma_{\mathrm{D}}}^{1}\left(\Omega ; \mathbb{R}^{3}\right), \quad \varphi\left(u^{\mathrm{e}}(t), v\right)=L(t)(v) \quad \text { for all } v \in H_{\Gamma_{\mathrm{D}}}^{1}\left(\Omega ; \mathbb{R}^{3}\right) \text { and all } t \in[0, T]
$$

where $H_{\Gamma_{\mathrm{D}}}^{1}\left(\Omega ; \mathbb{R}^{3}\right)$ denotes the closed subspace of $H^{1}\left(\Omega ; \mathbb{R}^{3}\right)$ consisting of the elements with zero trace on $\Gamma_{\mathrm{D}}$; hereafter, the notation $H_{\Gamma}^{1}\left(G ; \mathbb{R}^{3}\right)$ will be systematically used for any $G \subset \mathbb{R}^{3}, \Gamma \subset \partial G$. Furthermore,

$$
\begin{aligned}
& \varphi\left(v, v^{\prime}\right):=\int_{\Omega_{\varepsilon}} a e(v) \cdot e\left(v^{\prime}\right) \mathrm{d} x+\int_{B_{\varepsilon}} \mathrm{D} W_{\lambda, \mu}(e(v)) \cdot e\left(v^{\prime}\right) \mathrm{d} x \quad \text { for all } v, v^{\prime} \in H_{\Gamma_{\mathrm{D}}}^{1}\left(\Omega ; \mathbb{R}^{3}\right), \\
& L(t)(v):=\int_{\Gamma_{\mathrm{N}}} g(x, t) \cdot v(x) \mathrm{d} \mathcal{H}^{2}(x) \quad \text { for all } v \in H_{\Gamma_{\mathrm{D}}}^{1}\left(\Omega ; \mathbb{R}^{3}\right) \text { and all } t \in[0, T] .
\end{aligned}
$$

Since, for every fixed $t \in[0, T]$ the operator $g(t) \mapsto u^{\mathrm{e}}(t)$ is linear and continuous from $L^{2}\left(\Gamma_{\mathrm{N}} ; \mathbb{R}^{3}\right)$ to $H_{\Gamma_{\mathrm{D}}}^{1}\left(\Omega ; \mathbb{R}^{3}\right)$, we find that

$$
u^{\mathrm{e}} \in W^{2, \infty}\left(0, T ; H_{\Gamma_{\mathrm{D}}}^{1}\left(\Omega ; \mathbb{R}^{3}\right)\right)
$$

Given such $u^{\mathrm{e}}$, the remaining part $u^{\mathrm{r}}$ of $u$ can be obtained by solving the following evolutionary problem: Find $u^{\mathrm{r}}: \Omega \times[0, T] \rightarrow \mathbb{R}^{3}$ sufficiently smooth fulfilling (2.8f), the Cauchy conditions $u^{\mathrm{r}}(0)=u_{0}-u^{\mathrm{e}}(0)$ and $\partial_{t} u^{\mathrm{r}}(0)=v_{0}-\partial_{t} u^{\mathrm{e}}(0)$ in $\Omega$, and such that there exists $\xi: B_{\varepsilon} \rightarrow \mathbb{R}$ satisfying $\xi \in \partial \mathcal{D}\left(e\left(\partial_{t}\left(u^{\mathrm{r}}+u^{\mathrm{e}}\right)\right)\right)$ a.e. in $B_{\varepsilon}$ and

$$
\begin{aligned}
& \int_{\Omega} \gamma \partial_{t t}\left(u^{\mathrm{r}}(t)+u^{\mathrm{e}}(t)\right) \cdot v \mathrm{~d} x+\int_{\Omega_{\varepsilon}} a e\left(u^{\mathrm{r}}(t)\right) \cdot e(v) \mathrm{d} x+\int_{B_{\varepsilon}} \mathrm{D} W_{\lambda, \mu}\left(e\left(u^{\mathrm{r}}(t)\right)\right) \cdot e(v) \mathrm{d} x+b \int_{B_{\varepsilon}} \xi \cdot e(v) \mathrm{d} x \\
& =\int_{\Omega} f(t) \cdot v \mathrm{~d} x \quad \text { for all } v \in H_{\Gamma_{\mathrm{D}}}^{1}\left(\Omega ; \mathbb{R}^{3}\right) .
\end{aligned}
$$

In [18, the existence of a (unique) solution to the Cauchy problem for (3.5) was proved by reformulating it as an abstract evolutionary problem. Similarly arguing, we introduce the following problem

$$
\left\{\begin{array}{l}
\frac{\mathrm{d}}{\mathrm{d} t} \mathrm{X}(t)+\mathrm{AX}(t) \ni \mathrm{F}(t) \quad \text { in } \mathrm{H} \text { for a.a. } t \in(0, T) \\
\mathrm{X}(0)=\mathrm{X}_{0}
\end{array}\right.
$$

with $\mathrm{H}$ a (separable) Hilbert space (that will turn out to be the space of possible states with finite mechanical - i.e., (kinetic+strain) - energy), $\mathrm{A}: \mathrm{H} \rightrightarrows \mathrm{H}$ a maximal monotone (multivalued) operator, and $\mathrm{F} \in L^{1}(0, T ; \mathrm{H})$. More precisely, we consider the space

$$
\mathrm{H}:=H_{\Gamma_{\mathrm{D}}}^{1}\left(\Omega ; \mathbb{R}^{3}\right) \times L^{2}\left(\Omega ; \mathbb{R}^{3}\right)
$$

endowed with the following inner product and induced norm

$$
\begin{gathered}
\left(\mathrm{X}, \mathrm{X}^{\prime}\right):=\varphi\left(u, u^{\prime}\right)+k\left(v, v^{\prime}\right), \quad|\mathrm{X}|^{2}=(\mathrm{X}, \mathbf{X}) \quad \text { for all } \mathbf{X}=(u, v), \mathbf{X}^{\prime}=\left(u^{\prime}, v^{\prime}\right) \in \mathbf{H}, \\
\text { with } k\left(v, v^{\prime}\right):=\int_{\Omega} \gamma v v^{\prime} \mathrm{d} x \text { for all } v, v^{\prime} \in L^{2}\left(\Omega ; \mathbb{R}^{3}\right),
\end{gathered}
$$


and $\varphi$ defined in (3.3b). Observe that the norm induced by the bilinear form $\varphi$ is equivalent to the standard Sobolev norm on $H_{\Gamma_{\mathrm{D}}}^{1}\left(\Omega ; \mathbb{R}^{3}\right)$ by Korn's inequality. We introduce the operator $\mathrm{A}: \mathrm{H} \rightrightarrows \mathbf{H}$, with domain

$$
\begin{aligned}
& \mathrm{D}(\mathrm{A}):=\{\mathbf{X}=(u, v) \in \mathrm{H}:(1) v \in H_{\Gamma_{\mathrm{D}}}^{1}\left(\Omega ; \mathbb{R}^{3}\right) \\
&(2) \exists(w, \xi) \in L^{2}\left(\Omega ; \mathbb{R}^{3}\right) \times L^{2}\left(B_{\varepsilon} ; \mathbb{R}^{3}\right) \text { with } \xi \in \partial \mathcal{D}(e(v)) \text { a.e. in } B_{\varepsilon} \text {, s.t. } \\
&\left.k\left(w, v^{\prime}\right)+\varphi\left(u, v^{\prime}\right)+b \int_{B_{\varepsilon}} \xi \cdot e\left(v^{\prime}\right) \mathrm{d} x=0 \text { for all } v^{\prime} \in H_{\Gamma_{\mathrm{D}}}^{1}\left(\Omega ; \mathbb{R}^{3}\right)\right\},
\end{aligned}
$$

defined at every $\mathbf{X}=(u, v)$ by

$$
\mathrm{AX}:=\left(\begin{array}{c}
-v \\
0
\end{array}\right)+\left\{\left(\begin{array}{c}
0 \\
-w
\end{array}\right): w \text { as in }(3.8 \mathrm{a})(2)\right\}
$$

For later use, we recall the following result from [18, establishing a link between the equation defining the resolvent of A and the Euler-Lagrange equation for the minimization of the functional

$$
\mathcal{J}: H_{\Gamma_{\mathrm{D}}}^{1}\left(\Omega ; \mathbb{R}^{3}\right) \rightarrow \mathbb{R}, \quad \mathcal{J}(v):=\frac{1}{2} k(v, v)-k\left(\psi_{2}, v\right)+\frac{1}{2} \varphi(v, v)+\varphi\left(\psi_{1}, v\right)+b \int_{B_{\varepsilon}} \mathcal{D}(e(v)) \mathrm{d} x,
$$

with $\left(\psi_{1}, \psi_{2}\right) \in \mathbf{H}$ given.

Proposition 3.1 ([18], Prop. 3.1). The operator $\mathrm{A}$ is maximal monotone on $\mathrm{H}$ and its resolvent $(\mathrm{I}+\mathrm{A})^{-1}$ : $\mathbf{H} \rightarrow \mathbf{H}$ is given for all $\left(\psi_{1}, \psi_{2}\right) \in \mathbf{H}$ by $\mathbf{X}=(\mathbf{I}+\mathbf{A})^{-1}\left(\psi_{1}, \psi_{2}\right)$ if and only if

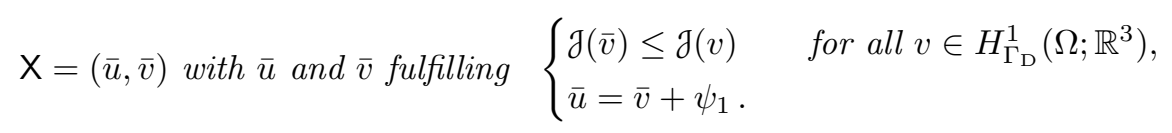

We now consider the Cauchy problem (3.6) with $\mathrm{H}$ from (3.7a), $\mathrm{A}$ from (3.8), and the data $\mathrm{F}$ and $\mathrm{X}_{0}$ given by

$$
\mathrm{F}=\left(-\partial_{t} u^{\mathrm{e}}, \frac{f}{\gamma}\right), \quad \mathrm{X}_{0}=\left(u_{0}, v_{0}\right)-\left(u^{\mathrm{e}}(0), 0\right) \text { such that } \mathrm{X}_{0} \in \mathrm{D}(\mathrm{A})
$$

We denote by $\mathrm{X}^{\mathrm{r}}=\left(u^{\mathrm{r}}, v^{\mathrm{r}}\right)$ the solution to (3.6), which exists, unique, in $W^{1, \infty}(0, T ; \mathbf{H})$ thanks to, e.g., 6 , Prop. 3.4]. By the careful definition of $\mathrm{H}, \mathrm{A}$, and of the data $\mathrm{F}$ and $\mathrm{X}_{0}$, it can be easily checked that $u^{\mathrm{r}}$ and that $v^{\mathrm{r}}=\partial_{t}\left(u^{\mathrm{r}}+u^{\mathrm{e}}\right)$ solve the Cauchy problem for (3.5).

Setting $u:=u^{\mathrm{r}}+u^{\mathrm{e}}$, we ultimately find the unique solution to Problem (P). This is summarized in the following result.

Theorem 3.2 (18, Thm. 3.1). Let the data $f, g$ comply with (3.1) and let $\left(u_{0}, v_{0}\right) \in\left(u^{\mathrm{e}}(0), 0\right)+\mathrm{D}(\mathrm{A})$. Then, the Cauchy problem (3.6) with $\mathrm{H}, \mathrm{A}$, and the data $\mathrm{F}$ and $\mathrm{X}_{0}$ from (3.7), (3.8), and (3.11), respectively, has a unique solution $\mathrm{X}^{\mathrm{r}}=\left(u^{\mathrm{r}}, v^{\mathrm{r}}\right) \in W^{1, \infty}(0, T ; \mathrm{H})$. Hence, there exists a unique $u \in W^{1, \infty}\left(0, T ; H_{\Gamma_{\mathrm{D}}}^{1}\left(\Omega ; \mathbb{R}^{3}\right)\right) \cap$ $W^{2, \infty}\left(0, T ; L^{2}\left(\Omega ; \mathbb{R}^{3}\right)\right)$, given by $u=u^{\mathrm{e}}+u^{\mathrm{r}}$, that satisfies (2.10) for all $v \in H_{\Gamma_{\mathrm{D}}}^{1}\left(\Omega ; \mathbb{R}^{3}\right)$ and for almost all $t \in(0, T)$ and complies with the Cauchy conditions (2.9).

\section{Asymptotic analysis}

In this section we address the asymptotic behavior of a sequence $\left(u_{n}\right)_{n}$ of solutions to Problems $\left(P_{n}\right)$ corresponding to a sequence $q_{n}=\left(\varepsilon_{n}, \lambda_{n}, \mu_{n}, b_{n}, \rho_{n}\right)$ of mechanical and geometrical parameters that satisfy the suitable conditions. As the overall density of the structure depends on the parameter $\rho_{n}$, we shall denote it by $\gamma_{n}$. The requirements in Hypothesis 4.1 below in particular reflect the fact that the adhesive layer has vanishing thickness and that the total mass of the adhesive layer remains strictly positive. 
Hypothesis 4.1. We suppose that

$$
\exists \lim _{n \rightarrow \infty} q_{n}=q_{\infty}=\left(\varepsilon_{\infty}, \lambda_{\infty}, \mu_{\infty}, b_{\infty}, \rho_{\infty}\right)
$$

such that

(1) $q_{\infty} \in\{0\} \times[0, \infty) \times[0, \infty) \times[0, \infty] \times\{\infty\}$;

(2) $\exists(\bar{\lambda}, \bar{\mu}) \in[0, \infty] \times[0, \infty]$ s.t. $(\bar{\lambda}, \bar{\mu})=\lim _{n \rightarrow \infty}\left(\frac{\lambda_{n}}{\varepsilon_{n}}, \frac{\mu_{n}}{\varepsilon_{n}}\right)$;

(3) $\lim _{n \rightarrow \infty} b_{n} \varepsilon_{n}=0$ and $\exists \bar{b} \in[0, \infty]$ s.t. $\bar{b}=\lim _{n \rightarrow \infty} \frac{b_{n}}{\varepsilon_{n}^{p-1}}$, where $p \in[1,2]$ is given as in (2.6);

(4) $\bar{\mu} \in(0, \infty]$ if $\min \left\{\mathcal{H}^{2}\left(\Gamma_{\mathrm{D}}^{ \pm}\right)\right\}=0$;

(5) $\limsup _{n \rightarrow \infty} \frac{\varepsilon_{n}^{2}}{\mu_{n}}<\infty$;

(6) $\exists \bar{\rho} \in(0, \infty)$ s.t. $\bar{\rho}=\lim _{n \rightarrow \infty} \rho_{n} \varepsilon_{n}$.

A comparison between Hypothesis 4.1 and the analogous assumption [18, (H2)] reveals that the only difference lies in condition (6) on the asymptotic behavior of the sequence $\left(\rho_{n}\right)_{n}$; condition (6) does indeed encompass the fact that the total mass of the adhesive layer is strictly positive, in the limit. As we will see, this will make the limiting problem significantly different from that considered in [18].

Actually, for the sake of simplicity and to highlight the main points in our analysis, in this paper we shall confine the discussion to the case in which $(\bar{\lambda}, \bar{\mu}) \in[0, \infty) \times(0, \infty)$. In the upcoming Section 4.1, with sort of heuristic arguments we propose a candidate PDE system for the description of the limiting behavior of the structure under Hypothesis 4.1 on the parameters $\left(q_{n}\right)_{n}$. As we will see, such a system may be somehow 'guessed', also based on the analysis previously performed in [18, cf. also [13].

In accordance with the approach developed in Sec. 3, the limiting system will be formulated as an evolutionary equation in a suitable Hilbert space, governed by a nonlinear maximal monotone operator. Next, in Sec. 4.2 we will carry out the asymptotic analysis via (a version of) Trotter's theory of approximation of semigroups on variable Hilbert spaces.

4.1. A candidate for the limiting behavior. The functional framework for the limiting problem is naturally obtained by studying the behavior of sequences $\left(\mathrm{X}_{n}\right)_{n}=\left(u_{n}, v_{n}\right)_{n}$ of possible mechanical states, uniformly bounded in the Hilbert spaces $\mathrm{H}_{n}$ from (3.7), namely

$$
\begin{aligned}
& \mathrm{H}_{n}:=H_{\Gamma_{\mathrm{D}}}^{1}\left(\Omega ; \mathbb{R}^{3}\right) \times L^{2}\left(\Omega ; \mathbb{R}^{3}\right) \text { endowed with the norms }|\cdot|_{n} \text { and the inner products } \\
& \left(\mathrm{X}, \mathrm{X}^{\prime}\right)_{n}:=\varphi_{n}\left(u, u^{\prime}\right)+k_{n}\left(v, v^{\prime}\right) \quad \text { for all } \mathrm{X}=(u, v), \mathrm{X}^{\prime}=\left(u^{\prime}, v^{\prime}\right) \in \mathrm{H} \quad \text { with } \\
& \begin{cases}\varphi_{n}\left(v, v^{\prime}\right):=\int_{\Omega_{\varepsilon}} a e(v) \cdot e\left(v^{\prime}\right) \mathrm{d} x+\int_{B_{\varepsilon_{n}}} \mathrm{D} W_{\lambda_{n}, \mu_{n}}(e(v)) \cdot e\left(v^{\prime}\right) \mathrm{d} x & \text { for all } v, v^{\prime} \in H_{\Gamma_{\mathrm{D}}}^{1}\left(\Omega ; \mathbb{R}^{3}\right), \\
k_{n}\left(v, v^{\prime}\right):=\int_{\Omega_{\varepsilon_{n}}} \rho^{*} v v^{\prime} \mathrm{d} x+\int_{B_{\varepsilon_{n}}} \rho_{n} v v^{\prime} \mathrm{d} x & \text { for all } v, v^{\prime} \in L^{2}\left(\Omega ; \mathbb{R}^{3}\right) .\end{cases}
\end{aligned}
$$

Therefore, for uniformly bounded mechanical states $\left(\mathrm{X}_{n}\right)_{n}=\left(u_{n}, v_{n}\right)_{n}$ the following estimates hold for a constant $C>0$ uniform w.r.t. $n \in \mathbb{N}$ :

$$
\begin{aligned}
& k_{n}\left(v_{n}, v_{n}\right)=\int_{\Omega_{\varepsilon_{n}}} \rho^{*}(x)\left|v_{n}\right|^{2} \mathrm{~d} x+\int_{B_{\varepsilon_{n}}} \rho_{n}\left|v_{n}\right|^{2} \mathrm{~d} x \leq C, \\
& \varphi_{n}\left(u_{n}, u_{n}\right)=\int_{\Omega_{\varepsilon_{n}}} a\left|e\left(u_{n}\right)\right|^{2} \mathrm{~d} x+\int_{B_{\varepsilon_{n}}} \mathrm{D} W_{\lambda_{n}, \mu_{n}}\left(e\left(u_{n}\right)\right) \cdot e\left(u_{n}\right) \mathrm{d} x \leq C .
\end{aligned}
$$

Let us now draw some conclusions from (4.3).

First of all, observe that, via a simple change of variables estimate (4.3a) may be rephrased as

$$
k_{n}\left(v_{n}, v_{n}\right)=\int_{\Omega_{\varepsilon_{n}}} \rho^{*}(x)\left|v_{n}\right|^{2} \mathrm{~d} x+\rho_{n} \varepsilon_{n} \int_{B}\left|\mathscr{S}_{\varepsilon_{n}}\left[v_{n}\right]\right|^{2} \mathrm{~d} x \leq C
$$


in terms of the operator $\mathscr{S}_{\varepsilon}$ that maps a function $v$ into the function $\mathscr{S}_{\varepsilon}[v]$ defined by

$$
\begin{aligned}
& \mathscr{S}_{\varepsilon}[v]\left(\hat{x}, x_{3}\right):=v\left(\hat{x}, \varepsilon x_{3}\right) \quad \text { for all } x=\left(\hat{x}, x_{3}\right) \in B:=S \times(-1,1) \\
& \text { and all measurable functions } v \text { on } B_{\varepsilon}=S \times(-\varepsilon, \varepsilon) .
\end{aligned}
$$

By virtue of Hypothesis 4.1(6) and condition (2.7b) on $\rho^{*}$, from the bound (4.4) we deduce that the pair

(1) $\left(\chi_{\Omega_{\varepsilon_{n}}} v_{n}, \mathscr{S}_{\varepsilon_{n}}\left[v_{n}\right]\right)_{n}$, up to a subsequence, weakly converge,

$$
\exists\left(v_{\Omega}, v_{B}\right) \in L^{2}\left(\Omega ; \mathbb{R}^{3}\right) \times L^{2}\left(B ; \mathbb{R}^{3}\right): \quad \chi_{\Omega_{\varepsilon_{n}}} v_{n} \rightarrow v_{\Omega} \text { in } L^{2}\left(\Omega ; \mathbb{R}^{3}\right), \mathscr{S}_{\varepsilon_{n}}\left[v_{n}\right] \rightarrow v_{B} \text { in } L^{2}\left(B ; \mathbb{R}^{3}\right) .
$$

Thus, we may describe the limiting kinetic state by two velocity fields $v_{\Omega}$ and $v_{B}$ that effectively represent the limiting behavior of the velocity in the adhering bodies and in the adhesive layer, respectively.

Secondly, we may deduce that there exists a pair $\left(u_{\Omega}, u_{B}\right) \in H_{\Gamma_{\mathrm{D}}}^{1}\left(\Omega \backslash S ; \mathbb{R}^{3}\right) \times L^{2}\left(B ; \mathbb{R}^{3}\right)$ such that, up to a subsequence,

(2) the functions $\chi_{\Omega_{\varepsilon_{n}}} e\left(u_{n}\right)$ converge weakly to $e\left(u_{\Omega}\right)$ in $L^{2}\left(\Omega ; \mathbb{R}^{3 \times 3}\right)$;

(3) the traces on $S_{\varepsilon_{n}}^{ \pm}$of $u_{n}$, considered as elements of $L^{2}\left(S ; \mathbb{R}^{3}\right)$, converge to the traces on $S$ of $u_{\Omega}^{ \pm}$(i.e., the restrictions of $u_{\Omega}$ to $\left.\Omega^{ \pm}\right)$strongly in $L^{2}\left(S ; \mathbb{R}^{3}\right)$;

(4) the functions $\mathscr{S}_{\varepsilon_{n}}\left[u_{n}\right]$ converge to $u_{B}$ weakly in $L^{2}\left(B ; \mathbb{R}^{3}\right)$.

Let us shortly justify properties (2)-(4). Indeed, following the lines of the proof of [18, Lemma 4.2], from the first two bounds in (4.3a) and (4.3b), via Korn's inequality and a standard diagonalization argument we infer that there exists $u_{\Omega} \in \bigcup_{\eta>0} H_{\Gamma_{\mathrm{D}}}^{1}\left(\Omega_{\eta} ; \mathbb{R}^{3}\right.$ ) (with $\Omega_{\eta}=\Omega \backslash \bar{B}_{\eta}$ ) such that, up to a subsequence, for all $\eta>0$ there holds $u_{n} \rightarrow u_{\Omega}$ weakly in $H^{1}\left(\Omega_{\eta} ; \mathbb{R}^{3}\right)$. In turn, there exists $e^{*} \in L^{2}\left(\Omega ; \mathbb{R}_{\text {sym }}^{3 \times 3}\right)$ such that $\chi_{\Omega_{\varepsilon_{n}}} e\left(u_{n}\right) \rightarrow e^{*}$ weakly in $L^{2}\left(\Omega ; \mathbb{R}_{\mathrm{sym}}^{3 \times 3}\right)$. Clearly, the restriction of $e^{*}$ to any $\Omega_{\eta}$ coincides with the restriction to $\Omega_{\eta}$ of $e\left(u_{\Omega}\right)$ (i.e. the symmetric part of the distributional gradient of $\left.u_{\Omega}\right)$. We thus conclude that $e\left(u_{\Omega}\right) \in L^{2}\left(\Omega ; \mathbb{R}_{\text {sym }}^{3 \times 3}\right)$ (whence $u_{\Omega} \in H_{\Gamma_{\mathrm{D}}}^{1}\left(\Omega \backslash S ; \mathbb{R}^{3}\right)$ ), and convergence (2) holds. Next, we repeat the very same arguments as in Step 2 of the proof of [18, Prop. 4.3], to conclude convergence (3). In order to check (4), we will first of all show that $\left(\mathscr{S}_{\varepsilon_{n}}\left[u_{n}\right]\right)_{n}$ is bounded in $L^{2}\left(B ; \mathbb{R}^{3}\right)$. To this end, we first of all employ the key inequality

$$
\begin{aligned}
& \exists C>0 \forall n \in \mathbb{N} \forall w \in H_{\Gamma_{\mathrm{D}}}^{1}\left(\Omega ; \mathbb{R}^{3}\right): \\
& \frac{1}{2} \int_{B_{\varepsilon_{n}}}|w|^{2} \mathrm{~d} x \leq \varepsilon_{n} \int_{S_{\varepsilon_{n}}}|w|^{2} \mathrm{~d} \hat{x}+C \varepsilon_{n}^{2} \int_{B_{\varepsilon_{n}}}|e(w)|^{2} \mathrm{~d} x+C \varepsilon_{n}^{2} \int_{\Omega_{\varepsilon_{n}}}|e(w)|^{2} \mathrm{~d} x,
\end{aligned}
$$

(cf. [18, (4.20)]), whence

$$
\frac{1}{2} \int_{B_{\varepsilon_{n}}}\left|u_{n}\right|^{2} \mathrm{~d} x \leq \varepsilon_{n} \int_{S_{\varepsilon_{n}}}\left|u_{n}\right|^{2} \mathrm{~d} x+C \varepsilon_{n}^{2}\left(\int_{\Omega_{\varepsilon_{n}}}\left|e\left(u_{n}\right)\right|^{2} \mathrm{~d} x+\int_{B_{\varepsilon_{n}}}\left|e\left(u_{n}\right)\right|^{2} \mathrm{~d} x\right) .
$$

Now, since

$$
\int_{S_{\varepsilon_{n}}}\left|u_{n}\right|^{2} \mathrm{~d} x \rightarrow \int_{S}\left(\left|\gamma_{S}\left(u_{\Omega}^{+}\right)\right|^{2}+\left|\gamma_{S}\left(u_{\Omega}^{-}\right)\right|^{2}\right) \mathrm{d} x
$$

we find that the first integral on the right-hand side is estimated by $C \varepsilon_{n}$. Furthermore, by (4.3b) we have that

$$
\int_{\Omega_{\varepsilon_{n}}}\left|e\left(u_{n}\right)\right|^{2} \mathrm{~d} x \leq C
$$

Finally,

$$
\varepsilon_{n}^{2} \int_{B_{\varepsilon_{n}}}\left|e\left(u_{n}\right)\right|^{2} \mathrm{~d} x \stackrel{(1)}{\leq} \frac{\varepsilon_{n}^{2}}{\mu_{n}} \int_{B_{\varepsilon_{n}}} \mathrm{D} W_{\lambda_{n}, \mu_{n}}\left(e\left(u_{n}\right)\right) \cdot e\left(u_{n}\right) \mathrm{d} x \stackrel{(2)}{\leq} C \varepsilon_{n},
$$


where (1) follows from (2.5) and (2) from the assumption that $\frac{\mu_{n}}{\varepsilon_{n}} \rightarrow \bar{\mu}>0$, cf. Hypothesis 4.1 (recall that we confine here our analysis to the case $\bar{\mu} \in(0,+\infty))$. All in all, we conclude that

$$
\varepsilon_{n} \int_{B}\left|\mathscr{S}_{\varepsilon_{n}}\left[u_{n}\right]\right|^{2} \mathrm{~d} x=\int_{B_{\varepsilon_{n}}}\left|u_{n}\right|^{2} \mathrm{~d} x \leq C \varepsilon_{n} \quad \text { whence } \quad \int_{B}\left|\mathscr{S}_{\varepsilon_{n}}\left[u_{n}\right]\right|^{2} \mathrm{~d} x \leq C .
$$

and convergence (4) follows.

Moreover, since

$$
\int_{B_{\varepsilon_{n}}}\left|e\left(u_{n}\right)\right|^{2} \mathrm{~d} x=\frac{1}{\varepsilon_{n}} \int_{B}\left|e\left(\varepsilon_{n}, \mathscr{S}_{\varepsilon_{n}}\left[u_{n}\right]\right)\right|^{2} \mathrm{~d} x
$$

where we have introduced the notation

$$
e(\varepsilon, w)_{i, j}:=\left\{\begin{array}{ll}
\varepsilon e(w)_{i, j} & \text { for } 1 \leq i, j \leq 2 \\
\frac{1}{2}\left(\varepsilon \partial_{i} w_{3}+\partial_{3} w_{i}\right) & \text { for } 1 \leq i \leq 2, j=3 \\
\partial_{3} w_{3} & \text { for } i=j=3
\end{array} \quad \text { for all } w \in H^{1}\left(B ; \mathbb{R}^{3}\right),\right.
$$

the convergence in the sense of distributions of $\mathscr{S}_{\varepsilon_{n}}\left[u_{n}\right]$ implies that

(5) $e\left(\varepsilon_{n}, \mathscr{S}_{\varepsilon_{n}}\left[u_{n}\right]\right)$ converge to $\partial_{3} u_{B} \otimes_{\mathrm{S}} e_{3}$ weakly in $L^{2}\left(B ; \mathbb{R}^{3} \times \mathbb{R}^{3}\right)$;

(6) $u_{B} \in H_{\partial_{3}}\left(B ; \mathbb{R}^{3}\right)$, with

$$
H_{\partial_{3}}\left(B ; \mathbb{R}^{3}\right):=\left\{u \in L^{2}\left(B ; \mathbb{R}^{3}\right): \partial_{3} u \in L^{2}\left(B ; \mathbb{R}^{3}\right)\right\}
$$

(7) the traces of $u_{B}$ on

$$
S^{+}:=S \times\{1\} \text { and } S^{-}:=S \times\{-1\},
$$

hereafter denoted by $\gamma_{S^{ \pm}}\left(u_{B}\right)$ and treated as elements of $L^{2}\left(S ; \mathbb{R}^{3}\right)$, coincide with the traces on $S$ of $u_{\Omega}^{ \pm}$, denoted by $\gamma_{S}\left(u_{\Omega}^{ \pm}\right)$.

Indeed, while items (5) \& (6) are obvious, (7) follows from observing that the traces of $u_{n}$ on $S_{\varepsilon_{n}}^{ \pm}$coincide with the traces of $\left(\mathscr{S}_{\varepsilon_{n}}\left[u_{n}\right]\right)^{ \pm}$on $S^{ \pm}$, and then taking the limit as $n \rightarrow \infty$.

In view of the above considerations, we thus expect that the Hilbert space of possible limiting states with finite energy will be

$$
\begin{aligned}
\mathrm{H} & :=\mathrm{U} \times \mathrm{V} \text { with } \\
\mathrm{U} & :=\left\{u=\left(u_{\Omega}, u_{B}\right) \in H^{1}\left(\Omega \backslash S ; \mathbb{R}^{3}\right) \times H_{\partial_{3}}\left(B ; \mathbb{R}^{3}\right): \gamma_{S}\left(u_{\Omega}^{ \pm}\right)=\gamma_{S^{ \pm}}\left(u_{B}\right)\right\}, \\
\mathrm{V} & :=\left\{v=\left(v_{\Omega}, v_{B}\right) \in L^{2}\left(\Omega ; \mathbb{R}^{3}\right) \times L^{2}\left(B ; \mathbb{R}^{3}\right)\right\},
\end{aligned}
$$

endowed with the inner product (and related norm)

$$
\left(\mathbf{X}, \mathbf{X}^{\prime}\right)=\varphi\left(u, u^{\prime}\right)+k\left(v, v^{\prime}\right), \quad|\mathbf{X}|^{2}:=\varphi(u, u)+k(v, v) \quad \text { for all } \mathbf{X}=(u, v), \mathbf{X}^{\prime}=\left(u^{\prime}, v^{\prime}\right) \in \mathbf{H},
$$

where

$$
\begin{aligned}
& \varphi\left(u, u^{\prime}\right):=\int_{\Omega \backslash S} a e\left(u_{\Omega}\right) \cdot e\left(u_{\Omega}^{\prime}\right) \mathrm{d} x+\int_{B} \mathrm{D} W_{\bar{\lambda}, \bar{\mu}}\left(\partial_{3} u_{B} \otimes_{\mathrm{S}} e_{3}\right) \cdot\left(\partial_{3} u_{B}^{\prime} \otimes_{\mathrm{S}} e_{3}\right) \mathrm{d} x, \\
& k\left(v, v^{\prime}\right)=\int_{\Omega} \rho^{*}\left|v_{\Omega}\right|^{2} \mathrm{~d} x+\bar{\rho} \int_{B}\left|v_{B}\right|^{2} \mathrm{~d} x .
\end{aligned}
$$

The limiting pseudopotential of dissipation is defined by

$$
\overline{\mathcal{D}}: L^{2}\left(B ; \mathbb{R}^{3}\right) \rightarrow[0, \infty] \quad \overline{\mathcal{D}}(q)= \begin{cases}\bar{b} \mathcal{D}^{\infty, p}\left(q \otimes_{\mathrm{S}} e_{3}\right) & \text { if } \bar{b} \in[0, \infty), \\ I_{\{0\}}\left(q \otimes_{\mathrm{S}} e_{3}\right) & \text { if } \bar{b}=\infty\end{cases}
$$

where $I_{\{0\}}$ is the indicator function of $\{0\}$ and

$$
\mathcal{D}^{\infty, p}\left(e^{\prime}\right)=\limsup _{t \rightarrow \infty} \frac{\mathcal{D}\left(t e^{\prime}\right)}{t^{p}} \quad \text { for all } e^{\prime} \in \mathbb{R}_{\mathrm{sym}}^{3 \times 3}
$$


and $p \in[1,2]$ is given as in (2.6). It is not difficult to check that

$$
I_{\{0\}}\left(q \otimes_{\mathrm{S}} e_{3}\right)<\infty \Leftrightarrow q=0 .
$$

In what follows, we shall assume

Hypothesis 4.2. We suppose that

$$
\exists \delta>0 \exists \theta \in(0, p) \forall e^{\prime} \in \mathbb{R}_{\mathrm{sym}}^{3 \times 3}: \quad\left|\mathcal{D}\left(e^{\prime}\right)-\mathcal{D}^{\infty, p}\left(e^{\prime}\right)\right| \leq \delta\left(1+\left|e^{\prime}\right|^{\theta}\right) .
$$

Hence, we can introduce the evolution operator $\mathrm{A}: \mathrm{H} \rightrightarrows \mathrm{H}$ with domain (cf. (4.14) $)$

$$
\begin{array}{r}
\mathrm{D}(\mathrm{A}):=\left\{\mathrm{X}=(u, v) \in \mathrm{H}:(1) v \in \mathrm{U} \text { and } \partial_{3} u_{B}=0 \text { if } \bar{b}=\infty,\right. \\
(2) \exists(w, \xi) \in \mathrm{V} \times L^{2}\left(B ; \mathbb{R}^{3}\right) \text { s.t. } \xi \in \partial \overline{\mathcal{D}}\left(\partial_{3} v_{B} \otimes_{\mathrm{S}} e_{3}\right) \text { a.e. in } B, \\
\left.k\left(w, v^{\prime}\right)+\varphi\left(u, v^{\prime}\right)+\int_{B} \xi \cdot\left(\partial_{3} v_{B}^{\prime} \otimes_{\mathrm{S}} e_{3}\right) \mathrm{d} x=0 \text { for all } v^{\prime} \in \mathrm{U} \text { as in (1) }\right\},
\end{array}
$$

defined at every $\mathrm{X}=(u, v)$ by

$$
\mathrm{AX}:=\left(\begin{array}{c}
-v \\
0
\end{array}\right)+\left\{\left(\begin{array}{c}
0 \\
-w
\end{array}\right): w \text { as in (4.16a) }(2)\right\} .
$$

With the same arguments as for the operator A from (3.8), it can be easily proved that A is maximal monotone in $\mathrm{H}$. Moreover, with the very same arguments as for Proposition 3.1. one can check that the resolvent of A satisfies

$$
\overline{\mathbf{X}}+\mathbf{A} \overline{\mathbf{X}} \ni\left(\psi_{1}, \psi_{2}\right) \quad \text { for every }\left(\psi_{1}, \psi_{2}\right) \in \mathbf{H}
$$

with $\overline{\mathrm{X}}=(\bar{u}, \bar{v})$, if and only if

$$
\left\{\begin{array}{l}
\mathcal{J}(\bar{v}) \leq \mathcal{J}(v) \quad \text { for all } v \in \mathrm{U} \\
\bar{u}=\bar{v}+\psi_{1}
\end{array}\right.
$$

with

$$
\mathcal{J}: U \rightarrow \mathbb{R}, \quad \mathcal{J}(v):=\frac{1}{2} k(v, v)-k\left(\psi_{2}, v\right)+\frac{1}{2} \varphi(v, v)+\varphi\left(\psi_{1}, v\right)+\int_{B} \overline{\mathcal{D}}\left(\partial_{3} v_{B} \otimes_{\mathrm{S}} e_{3}\right) \mathrm{d} x
$$

By arguing as in Section 3, the expected limit of the sequence $\left(u_{n}:=u_{n}^{\mathrm{e}}+u_{n}^{\mathrm{r}}\right)_{n}$ (cf. (3.2) ) of the solutions to Problems $\left(P_{n}\right)$ will be the sum of some $u^{\mathrm{e}}$, solution to a limiting 'stationary' problem and some $u^{\mathrm{r}}$, solution to a limiting evolutionary problem. More precisely, we will have that $u^{\mathrm{e}}=\left(u_{\Omega}^{\mathrm{e}}, u_{B}^{\mathrm{e}}\right) \in W^{2, \infty}(0, T ; \mathrm{U})$, with $u_{B}^{\mathrm{e}}$ affine in $x_{3}$ (cf. Remark 4.4 ahead) is the unique solution to

$$
\varphi\left(u^{\mathrm{e}}(t), v\right)=L(t)(v) \quad \text { for all } v \in \mathrm{U} \text { for all } t \in[0, T],
$$

with $\varphi$ from (4.12b) and $L$ from (3.3c). Instead, $u^{\mathrm{r}}$ is the first component of the solution $\mathrm{X}^{\mathrm{r}}=\left(u^{\mathrm{r}}, v^{\mathrm{r}}\right)$ to the following abstract problem

$$
\left\{\begin{array}{l}
\frac{\mathrm{d}}{\mathrm{d} t} \mathrm{X}(t)+\mathrm{AX}(t) \ni \mathrm{F}(t) \quad \text { in } \mathrm{H} \text { for a.a. } t \in(0, T) \\
\mathrm{X}(0)=\mathrm{X}_{0}
\end{array}\right.
$$

with $\mathrm{X}_{0}$ specified later on and $\mathrm{F}$ given by

$$
\mathrm{F}=\left(-\partial_{t} u^{\mathrm{e}},\left(\frac{f}{\rho^{*}}, 0\right)\right)
$$

We postpone to Section 5 some comments on the variational formulation of the initial-boundary value problem (4.19), cf. (5.1) and (5.4) ahead.

In the same way as for the Cauchy problem (3.6) with the forcing term F from (3.11), the results in [6, Sec. III.2]) yield 
Proposition 4.3. If $\mathrm{X}_{0} \in \mathrm{D}(\mathrm{A})$ and if $(f, g)$ satisfy (3.1), then (4.19) has a unique solution $\mathrm{X}^{\mathrm{r}}=\left(u^{\mathrm{r}}, v^{\mathrm{r}}\right) \in$ $W^{1, \infty}(0, T ; \mathrm{H})$.

We set

$$
\mathrm{X}^{\mathrm{e}}=\left(u^{\mathrm{e}}, 0\right), \quad \mathrm{X}=\mathrm{X}^{\mathrm{r}}+\mathrm{X}^{\mathrm{e}}
$$

We are now in a position to outline our argument for proving the convergence of the sequence $\left(u_{n}=u_{n}^{\mathrm{e}}+u_{n}^{\mathrm{r}}\right)_{n}$ to $u=u^{\mathrm{e}}+u^{\mathrm{r}}$ :

(1) the convergence of $u_{n}^{\mathrm{e}}$ to $u^{\mathrm{e}}$ will be obtained in Proposition 4.11 ahead, as part of the proof of

(2) the convergence of $u_{n}^{\mathrm{r}}$ to $u^{\mathrm{r}}$, stated in Theorem 4.15]ahead. For proving it, we will resort to a nonlinear version of Trotter's theory of approximation of semigroups acting on variable spaces, as developed in the Appendix of [20]. The need for such a theory is motivated by the fact that the functions $u_{n}^{\mathrm{r}}$ and $u^{\mathrm{r}}$ do not belong to the same space.

The proof of Theorem 4.15 will be carried out throughout Section 4.2

We now conclude this section by specifying the structure of the solution $u^{\mathrm{e}}=\left(u_{\Omega}^{\mathrm{e}}, u_{B}^{\mathrm{e}}\right)$ to the limit stationary problem (4.18). In particular, we will show that $u_{B}^{\mathrm{e}}$ is affine in $x_{3}$.

Remark 4.4. Let $u^{\mathrm{e}}=\left(u_{\Omega}^{\mathrm{e}}, u_{B}^{\mathrm{e}}\right) \in W^{2, \infty}(0, T ; \mathrm{U})$ be the unique solution to (4.18) with $\varphi$ from (4.12b) and $L$ from (3.3c). Then $u^{\mathrm{e}}$ satisfies

$$
\begin{aligned}
& \int_{\Omega \backslash S} a e\left(u_{\Omega}^{\mathrm{e}}\right)(t) \cdot e\left(v_{\Omega}\right) \mathrm{d} x+\int_{B} \mathrm{D} W_{\bar{\lambda}, \bar{\mu}}\left(\partial_{3} u_{B}^{\mathrm{e}}(t) \otimes_{\mathrm{S}} e_{3}\right) \cdot\left(\partial_{3} v_{B} \otimes_{\mathrm{S}} e_{3}\right) \mathrm{d} x \\
& =\int_{\Gamma_{\mathrm{N}}} g(t) \cdot v_{\Omega} \mathrm{d} \mathcal{H}^{2}(x) \quad \text { for all } v=\left(v_{\Omega}, v_{B}\right) \in \mathrm{U} \text { and for all } t \in[0, T] .
\end{aligned}
$$

Choosing now $v_{\Omega}=0$ and $v_{B}$ as an arbitrary test function $\varphi \in \mathrm{C}_{\mathrm{c}}^{\infty}\left(B ; \mathbb{R}^{3}\right)$ leads to

$$
\int_{B}\left(\bar{\mu} \partial_{3}\left(u_{B}^{\mathrm{e}}\right)_{1} \partial_{3} \varphi_{1}+\bar{\mu} \partial_{3}\left(u_{B}^{\mathrm{e}}\right)_{2} \partial_{3} \varphi_{2}+(\bar{\lambda}+2 \bar{\mu}) \partial_{3}\left(u_{B}^{\mathrm{e}}\right)_{3} \partial_{3} \varphi_{3}\right) \mathrm{d} x=0 \quad \text { for all } \varphi \in \mathrm{C}_{\mathrm{c}}^{\infty}\left(B ; \mathbb{R}^{3}\right),
$$

where $\partial_{3}\left(u_{B}^{\mathrm{e}}\right)_{i}$ denotes the $i$ th-component of the vector $\partial_{3} u_{B}^{\mathrm{e}}$. This implies that the function $x_{3} \mapsto u_{B}^{\mathrm{e}}\left(\hat{x}, x_{3}, t\right)$ is affine.

4.2. Convergence. Throughout this section, we will implicitly assume the validity of Hypotheses 4.1 and 4.2 , and of conditions (3.1) on the problem data $f$ and $g$. In particular, we shall omit to invoke these assumptions in all of the statements of the various results, with the exception of Theorem 4.15

In the next subsection we shortly recapitulate the basics of the result from Trotter theory that we shall use to prove Theorem 4.15.

4.2.1. Recaps on Trotter's theory of approximation of semigroups. Let us first fix some preliminary definitions. We consider

a sequence $\left(\mathrm{H}_{n}\right)_{n}$ of Hilbert spaces, with inner products $(\cdot, \cdot)_{n}$ and norms $|\cdot|_{n}$,

and a 'limiting' Hilbert space $\mathrm{H}$, such that for every $n \in \mathbb{N}$ there is defined an operator $\mathrm{P}_{n}: \mathrm{H} \rightarrow \mathrm{H}_{n}$, linear and continuous, fulfilling the following properties:

There exists $C>0$ such that for every $n \in \mathbb{N}$ and $\mathrm{X} \in \mathrm{H}$ there holds $\left|\mathrm{P}_{n} \mathrm{X}\right|_{n} \leq C|\mathrm{X}|$;

For every $\mathrm{X} \in \mathrm{H}$ there holds $\lim _{n \rightarrow \infty}\left|\mathrm{P}_{n} \mathrm{X}\right|_{n}=|\mathrm{X}|$.

Next, for a given sequence $\left(\mathrm{X}_{n}\right)_{n}$ with $\mathrm{X}_{n} \in \mathrm{H}_{n}$ for every $n \in \mathbb{N}$, we will say that

$$
\left(\mathrm{X}_{n}\right)_{n} \text { converge to } \mathrm{X} \in \mathrm{H} \text { in the sense of Trotter if } \lim _{n \rightarrow \infty}\left|\mathrm{P}_{n} \mathrm{X}-\mathrm{X}_{n}\right|_{n}=0 .
$$

We are now in a position to recall the result from [20] needed for our analysis. 
Theorem 4.5 (20], Thm. 5). Suppose that the Hilbert spaces $\mathrm{H}_{n}, \mathrm{H}$ fulfill (4.23). Let $\mathrm{A}_{n}: \mathrm{H}_{n} \rightrightarrows \mathrm{H}_{n}$, $\mathrm{A}: \mathrm{H} \rightrightarrows \mathrm{H}$ be maximal monotone operators, let $\mathrm{F}_{n} \in L^{1}\left(0, T ; \mathrm{H}_{n}\right), \mathrm{F} \in L^{1}(0, T ; \mathrm{H})$, and let $\mathrm{X}_{n}^{0} \in \overline{\mathrm{D}\left(\mathrm{A}_{n}\right)}$, $\mathrm{X}^{0} \in \overline{\mathrm{D}(\mathrm{A})}$. Let $\left(\mathrm{X}_{n}\right)_{n}, \mathrm{X}$ be the weak solutions to the Cauchy problems

$$
\begin{aligned}
& \left\{\begin{array}{l}
\frac{\mathrm{d}}{\mathrm{d} t} \mathrm{X}_{n}(t)+\mathrm{A}_{n} \mathrm{X}_{n}(t) \ni \mathrm{F}_{n}(t) \quad \text { in } \mathrm{H}_{n} \text { for a.a. } t \in(0, T), \\
\mathrm{X}_{n}(0)=\mathrm{X}_{n}^{0},
\end{array}\right. \\
& \left\{\begin{array}{l}
\frac{\mathrm{d}}{\mathrm{d} t} \mathrm{X}(t)+\mathrm{AX}(t) \ni \mathrm{F}(t) \\
\mathrm{X}(0)=\mathrm{X}^{0} .
\end{array}\right.
\end{aligned}
$$

If

$$
\lim _{n \rightarrow \infty}\left|\mathrm{P}_{n}\left(\mathrm{X}^{0}\right)-\mathrm{X}_{n}^{0}\right|_{n}=0 \quad \text { and } \quad \lim _{n \rightarrow \infty} \int_{0}^{T}\left|\mathrm{P}_{n}(\mathrm{~F}(t))-\mathrm{F}_{n}(t)\right|_{n} \mathrm{~d} t=0
$$

and if for every $\lambda \geq 0$ and $\mathrm{X} \in \mathrm{H}$ we have that

the sequence $\left(\left(\mathrm{I}+\lambda \mathrm{A}_{n}\right)^{-1}\left(\mathrm{P}_{n}(\mathrm{X})\right)\right)_{n}$ converge in the sense of Trotter to $(\mathrm{I}+\lambda \mathrm{A})^{-1}(\mathrm{X})$ as $n \rightarrow \infty$,

(where we denote by the same symbol the identity operators $\mathbf{I}: \mathrm{H}_{n} \rightarrow \mathrm{H}_{n}$ and $\left.\mathrm{I}: \mathrm{H} \rightarrow \mathrm{H}\right)$, then $\left(\mathrm{X}_{n}\right)_{n}$ converge to $\mathrm{X}$ in the sense of Trotter uniformly on $[0, T]$, namely

$$
\lim _{n \rightarrow \infty} \sup _{t \in[0, T]}\left|\mathrm{P}_{n}(\mathrm{X}(\mathrm{t}))-\mathrm{X}_{n}(t)\right|_{n}=0 .
$$

4.2.2. Setting up Trotter's theory for our problem. In what follows, we establish the setup in which we shall apply Thm. 4.5. We consider the Hilbert spaces $\mathrm{H}_{n}$ from (4.2), while the 'limiting' Hilbert space $\mathrm{H}$ is given by (4.11), with the inner product from (4.12). Now, in order to apply Thm. 4.5 we have to introduce a linear continuous operator $\mathrm{P}_{n}: \mathrm{H} \rightarrow \mathrm{H}_{n}$ that with any element $\mathrm{X} \in \mathrm{H}$ associates a suitable representative $\mathrm{P}_{n}(\mathrm{X}) \in \mathrm{H}_{n}$. Therefore, the operator $\mathrm{P}_{n}: \mathrm{H} \rightarrow \mathrm{H}_{n}$ shall have the form

$$
\mathrm{P}_{n}(\mathrm{X})=\mathrm{P}_{n}\left(\left(u_{\Omega}, u_{B}\right) ;\left(v_{\Omega}, v_{B}\right)\right)=\left(\mathrm{P}_{n}^{u}\left(u_{\Omega}, u_{B}\right) ; \mathrm{P}_{n}^{v}\left(v_{\Omega}, v_{B}\right)\right) .
$$

The choice for the operator $\mathrm{P}_{n}^{v}: \mathrm{V} \rightarrow L^{2}\left(\Omega ; \mathbb{R}^{3}\right)$ (with $\mathrm{V}$ from (4.11c)) is guided by the idea of describing the limiting state in terms of two velocity fields, namely we set

$$
\mathrm{P}_{n}^{v}\left(v_{\Omega}, v_{B}\right):=\chi_{\Omega_{\varepsilon_{n}}} v_{\Omega}+\left(1-\chi_{\Omega_{\varepsilon_{n}}}\right)\left(\mathscr{S}_{\varepsilon_{n}}\right)^{-1}\left[v_{B}\right]
$$

The choice for $\mathrm{P}_{n}^{u}$, specified in (4.38) below, reflects how a field like $u_{B}$ may be involved in the asymptotic behavior of $\left(u_{n}\right)_{n}$. Indeed, first of all we consider the unique function $u_{B}^{n}$ satisfying

$$
\begin{gathered}
u_{B}^{n} \in H^{1}\left(B_{\varepsilon_{n}} ; \mathbb{R}^{3}\right) \text { with } u_{B}^{n}\left(\hat{x}, \pm \varepsilon_{n}\right)=u_{\Omega}^{ \pm}(\hat{x}, 0) \text { for a.a. } \hat{x} \in S \text { and } \\
\int_{B_{\varepsilon_{n}}} \mathrm{D} W_{\lambda_{n}, \mu_{n}}\left(e\left(u_{B}^{n}\right)\right) \cdot e(\varphi) \mathrm{d} x=\int_{B} \mathrm{D} W_{\bar{\lambda}, \bar{\mu}}\left(\partial_{3} u_{B} \otimes_{\mathrm{S}} e_{3}\right) \cdot e\left(\varepsilon_{n}, \mathscr{S}_{\varepsilon_{n}}[\varphi]\right) \mathrm{d} x \quad \text { for all } \varphi \in H_{S_{\varepsilon_{n}}^{+} \cup S_{\varepsilon_{n}}^{-}}^{1}\left(B_{\varepsilon_{n}} ; \mathbb{R}^{3}\right),
\end{gathered}
$$

where $H_{S_{\varepsilon_{n}}^{+} \cup S_{\varepsilon_{n}}^{-}}^{1}\left(B_{\varepsilon_{n}} ; \mathbb{R}^{3}\right)$ denotes the closed subspace of $H^{1}\left(B_{\varepsilon_{n}} ; \mathbb{R}^{3}\right)$ consisting of the functions with null trace on $S_{\varepsilon_{n}}^{+} \cup S_{\varepsilon_{n}}^{-}$. From the functions $u_{B}^{n}$ we then derive functions defined on $B=S \times(-1,1)$ by resorting to the operator $\mathscr{S}_{\varepsilon_{n}}$. Namely, we set

$$
u_{B, n}:=\mathscr{S}_{\varepsilon_{n}}\left[u_{B}^{n}\right] .
$$

From (4.31) we deduce that the functions $u_{B, n}$ fulfill (recall that $\gamma_{S^{ \pm}}$denote the traces on $S^{ \pm}=S \times\{ \pm 1\}$, cf. (4.10)

$$
\begin{gathered}
u_{B, n} \in H^{1}\left(B ; \mathbb{R}^{3}\right) \text { with } \gamma_{S^{ \pm}}\left(u_{B, n}\right)=\gamma_{S^{ \pm}}\left(u_{B}\right) \text { and } \\
\frac{1}{\varepsilon_{n}} \int_{B} \mathrm{D} W_{\lambda_{n}, \mu_{n}}\left(e\left(\varepsilon_{n}, u_{B, n}\right)\right) \cdot e\left(\varepsilon_{n}, \psi\right) \mathrm{d} x=\int_{B} \mathrm{D} W_{\bar{\lambda}, \bar{\mu}}\left(\partial_{3} u_{B} \otimes_{S} e_{3}\right) \cdot e\left(\varepsilon_{n}, \psi\right) \mathrm{d} x \quad \text { for all } \psi \in H_{S^{+} \cup S^{-}}^{1}\left(B ; \mathbb{R}^{3}\right) .
\end{gathered}
$$


The functions $u_{B, n}$ will enter into the definition of $\mathrm{P}_{n}^{u}$. Before specifying in which way, however, let us gain further insight into the properties of the sequence $\left(u_{B, n}\right)_{n}$ in the following result, where we are using the notation $\widehat{u}_{B, n}$ for the first two components of the function $u_{B, n}$, cf. Notation 1.1 .

Lemma 4.6. The following properties hold:

(1) the sequence $\left(\widehat{u}_{B, n}\right)_{n}$ converge weakly to $\widehat{u}_{B}$ in $L^{2}\left(B ; \mathbb{R}^{2}\right)$;

(2) the sequence $\left(\left(u_{B, n}\right)_{3}, e\left(\varepsilon_{n}, u_{B, n}\right)\right)_{n}$ converge strongly to $\left(u_{B 3}, \partial_{3} u_{B} \otimes_{\mathrm{S}} e_{3}\right)$ in $H_{\partial_{3}}(B) \times L^{2}\left(B ; \mathbb{R}_{\mathrm{sym}}^{3 \times 3}\right)$ (with the space $H_{\partial_{3}}(B)$ defined analogously as $H_{\partial_{3}}\left(B ; \mathbb{R}^{3}\right)$, cf. (4.9));

(3) if $u_{B}$ belongs to $H^{1}\left(B ; \mathbb{R}^{3}\right)$, then $\left(u_{B, n}\right)_{n}$ converge strongly to $u_{B}$ in $L^{2}\left(B ; \mathbb{R}^{3}\right)$.

Proof. Let $u_{B}^{*} \in H^{1}\left(B ; \mathbb{R}^{3}\right)$ fulfill $\gamma_{S^{ \pm}}\left(u_{B}^{*}\right)=\gamma_{S^{ \pm}}\left(u_{B}\right)$. We plug in (4.33) the test function $\psi=u_{B, n}-u_{B}^{*}$, thus obtaining

$$
\frac{1}{\varepsilon_{n}} \int_{B} \mathrm{D} W_{\lambda_{n}, \mu_{n}}\left(e\left(\varepsilon_{n}, u_{B, n}\right)\right) \cdot e\left(\varepsilon_{n}, u_{B, n}-u_{B}^{*}\right) \mathrm{d} x=\int_{B} \mathrm{D} W_{\bar{\lambda}, \bar{\mu}}\left(\partial_{3} u_{B} \otimes_{\mathrm{S}} e_{3}\right) \cdot e\left(\varepsilon_{n}, u_{B, n}-u_{B}^{*}\right) \mathrm{d} x .
$$

Combining the estimate $\frac{1}{\varepsilon_{n}} \mathrm{D} W_{\lambda_{n} \mu_{n}}(e) \cdot e \geq \frac{\mu_{n}}{\varepsilon_{n}}|e|^{2}$ (cf.(2.5) $)$ with the fact that $\frac{\mu_{n}}{\varepsilon_{n}} \rightarrow \bar{\mu}>0$ by Hypothesis 4.1 , from (4.34) we easily deduce that

$$
\exists C>0 \forall n \in \mathbb{N}: \quad \frac{\bar{\mu}}{2} \int_{B}\left|e\left(\varepsilon_{n}, u_{B, n}\right)\right|^{2} \mathrm{~d} x \leq C\left(1+\left\|e\left(\varepsilon_{n}, u_{B, n}\right)\right\|_{L^{2}\left(B ; \mathbb{R}_{\mathrm{sym}}^{3}\right)}\right) .
$$

This implies that the sequence $\left(e\left(\varepsilon_{n}, u_{B, n}\right)\right)_{n}$ is bounded in $L^{2}\left(B ; \mathbb{R}_{\mathrm{sym}}^{3}\right)$. We will use this to conclude that $\left(u_{B, n}\right)_{n}$ is bounded in $L^{2}\left(B ; \mathbb{R}^{3}\right)$ via a Poincaré-type estimate, namely

$$
\exists C>0 \forall n \in \mathbb{N} \forall z \in H_{S^{+} \cup S^{-}}^{1}\left(B ; \mathbb{R}^{3}\right): \quad \int_{B}|z|^{2} \mathrm{~d} x \leq C \int_{B}\left|e\left(\varepsilon_{n}, z\right)\right|^{2} \mathrm{~d} x .
$$

We deduce (4.35) from estimate (4.7), written for the function

$$
w:= \begin{cases}0 & \text { in } \Omega_{\varepsilon_{n}}, \\ \mathscr{S}_{\varepsilon_{n}}^{-1}[z] & \text { in } B_{\varepsilon_{n}}\end{cases}
$$

(observe that $\mathscr{S}_{\varepsilon_{n}}^{-1}[z] \in H^{1}\left(B_{\varepsilon_{n}} ; \mathbb{R}^{3}\right)$ with $\left.\gamma_{S_{\varepsilon_{n}}^{ \pm}}\left(\mathscr{S}_{\varepsilon_{n}}^{-1}[z]\right)=0\right)$. Then,

$$
\begin{aligned}
\int_{B}|z|^{2} \mathrm{~d} x=\int_{B}\left|\mathscr{S}_{\varepsilon_{n}}\left[\mathscr{S}_{\varepsilon_{n}}^{-1}[z]\right]\right|^{2} \mathrm{~d} x & =\frac{1}{\varepsilon_{n}} \int_{B_{\varepsilon_{n}}}\left|\mathscr{S}_{\varepsilon_{n}}^{-1}[z]\right|^{2} \mathrm{~d} x \\
& \stackrel{(1)}{\leq} \frac{1}{\varepsilon_{n}}\left(2 \varepsilon_{n} \int_{S_{\varepsilon_{n}}}\left|\mathscr{S}_{\varepsilon_{n}}^{-1}[z]\right|^{2} \mathrm{~d} x+C \varepsilon_{n}^{2} \int_{B_{\varepsilon_{n}}}\left|e\left(\mathscr{S}_{\varepsilon_{n}}^{-1}[z]\right)\right|^{2} \mathrm{~d} x+0\right) \\
& \stackrel{(2)}{=} C \varepsilon_{n} \int_{B_{\varepsilon_{n}}}\left|e\left(\mathscr{S}_{\varepsilon_{n}}^{-1}[z]\right)\right|^{2} \mathrm{~d} x \\
& \stackrel{(3)}{=} C \int_{B}\left|e\left(\varepsilon_{n}, \mathscr{S}_{\varepsilon_{n}}\left[\mathscr{S}_{\varepsilon_{n}}^{-1}[z]\right]\right)\right|^{2} \mathrm{~d} x=C \int_{B}\left|e\left(\varepsilon_{n}, z\right)\right|^{2} \mathrm{~d} x,
\end{aligned}
$$

where (1) follows from (4.7), (2) from the fact that $\gamma_{S_{\varepsilon_{n}}^{ \pm}}\left(\mathscr{S}_{\varepsilon_{n}}^{-1}[z]\right)=0$, and (3) from (4.8). Next, choosing $z=u_{B, n}-u_{B}^{*}$ in (4.35) we conclude that

$$
\int_{B}\left|u_{B, n}-u_{B}^{*}\right|^{2} \mathrm{~d} x \leq C \int_{B}\left|e\left(\varepsilon_{n}, u_{B, n}-u_{B}^{*}\right)\right|^{2} \mathrm{~d} x .
$$

Since $\left(e\left(\varepsilon_{n}, u_{B, n}\right)\right)_{n}$ is bounded in $L^{2}\left(B ; \mathbb{R}_{\mathrm{sym}}^{3}\right)$, we infer that $\left(u_{B, n}\right)_{n}$ is bounded in $L^{2}\left(B ; \mathbb{R}^{3}\right)$. Therefore, up to a (not relabelled) subsequence, the functions $\left(u_{B, n}, e\left(\varepsilon_{n}, u_{B, n}\right)\right)_{n}$ weakly converge to a pair $\left(\bar{u}_{B}, \partial_{3} \bar{u}_{B} \otimes_{\mathrm{S}} e_{3}\right)$, 
where the identification of the weak limit of $\left(e\left(\varepsilon_{n}, u_{B, n}\right)\right)_{n}$ follows from a distributional convergence argument. We are then in a position to pass to the limit in (4.33) and thus deduce that the function $\bar{u}_{B}$ fulfills

$\bar{u}_{B} \in H_{\partial_{3}}^{1}\left(B ; \mathbb{R}^{3}\right)$ with $\gamma_{S^{ \pm}}\left(\bar{u}_{B}\right)=\gamma_{S^{ \pm}}\left(u_{B}\right)$ and for all $\psi \in H_{\partial_{3}}^{1}\left(B ; \mathbb{R}^{3}\right)$ with $\gamma_{S^{ \pm}}(\psi)=0$ there holds

$$
\int_{B} \mathrm{D} W_{\bar{\lambda}, \bar{\mu}}\left(\partial_{3} \bar{u}_{B} \otimes_{\mathrm{S}} e_{3}\right) \cdot\left(\partial_{3} \psi \otimes_{\mathrm{S}} e_{3}\right) \mathrm{d} x=\int_{B} \mathrm{D} W_{\bar{\lambda}, \bar{\mu}}\left(\partial_{3} u_{B} \otimes_{\mathrm{S}} e_{3}\right) \cdot\left(\partial_{3} \psi \otimes_{\mathrm{S}} e_{3}\right) \mathrm{d} x
$$

Therefore, $\partial_{3}\left(\bar{u}_{B}-u_{B}\right)=0$ and, since $\gamma_{S^{ \pm}}\left(\bar{u}_{B}\right)=\gamma_{S^{ \pm}}\left(u_{B}\right)$, we ultimately have that $\bar{u}_{B}=u_{B}$. Having uniquely identified the limit we eventually gain convergence along the whole sequence $\left(\varepsilon_{n}\right)_{n}$. We thus conclude claim (1).

Eventually, (4.34) implies

$$
\begin{aligned}
\lim _{n \rightarrow \infty} \int_{B} 2 W_{\bar{\lambda}, \bar{\mu}}\left(e\left(\varepsilon_{n}, u_{B, n}\right)\right) \mathrm{d} x & =\lim _{n \rightarrow \infty} \int_{B} \mathrm{D} W_{\bar{\lambda}, \bar{\mu}}\left(e\left(\varepsilon_{n}, u_{B, n}\right)\right) \cdot e\left(\varepsilon_{n}, u_{B, n}\right) \mathrm{d} x \\
& =\lim _{n \rightarrow \infty} \frac{1}{\varepsilon_{n}} \int_{B} \mathrm{D} W_{\lambda_{n}, \mu_{n}}\left(e\left(\varepsilon_{n}, u_{B, n}\right)\right) \cdot e\left(\varepsilon_{n}, u_{B, n}\right) \mathrm{d} x \\
& =\int_{B} \mathrm{D} W_{\bar{\lambda}, \bar{\mu}}\left(\partial_{3} u_{B} \otimes_{\mathrm{S}} e_{3}\right) \cdot\left(\partial_{3} u_{B} \otimes_{\mathrm{S}} e_{3}\right) \mathrm{d} x \\
& =\int_{B} 2 W_{\bar{\lambda}, \bar{\mu}}\left(\partial_{3} u_{B} \otimes_{\mathrm{S}} e_{3}\right) \mathrm{d} x
\end{aligned}
$$

Since the functional $q \mapsto\left(\int_{B} W_{\bar{\lambda}, \bar{\mu}}(q) \mathrm{d} x\right)^{1 / 2}$ induces a norm equivalent to the usual one on $L^{2}\left(B ; \mathbb{R}_{\mathrm{sym}}^{3 \times 3}\right)$, we thus deduce that $e\left(\varepsilon_{n}, u_{B, n}\right)$ converge to $\partial_{3} u_{B} \otimes_{\mathrm{S}} e_{3}$ strongly in $L^{2}\left(B ; \mathbb{R}_{\mathrm{sym}}^{3 \times 3}\right)$ and then $\left(u_{B, n}\right)_{3} \rightarrow\left(u_{B}\right)_{3}$ strongly in $H_{\partial_{3}}^{1}(B)$. This gives claim (2).

Finally, suppose that $u_{B} \in H^{1}\left(B ; \mathbb{R}^{3}\right)$. The analogue of (4.36), i.e. $\int_{B}\left|u_{B, n}-u_{B}\right|^{2} \mathrm{~d} x \leq C \int_{B}\left|e\left(\varepsilon_{n}, u_{B, n}-u_{B}\right)\right|^{2} \mathrm{~d} x$, combined with claim (2), yields that $u_{B, n} \rightarrow u_{B}$ strongly in $L^{2}\left(B ; \mathbb{R}^{3}\right)$. This concludes the proof.

We define the operator $\mathrm{P}_{n}^{u}: \mathrm{U} \rightarrow H_{\Gamma_{\mathrm{D}}}^{1}\left(\Omega ; \mathbb{R}^{3}\right)$ (with $\mathrm{U}$ from (4.11b) by

$$
\mathrm{P}_{n}^{u}\left(u_{\Omega}, u_{B}\right)(x):= \begin{cases}\left(\mathscr{S}_{\varepsilon_{n}}\right)^{-1}\left[u_{B, n}\right](x)=u_{B}^{n}(x) & \text { if } x \in B_{\varepsilon_{n}}, \\ \xi\left(x_{3}\right) u_{\Omega}\left(\hat{x}, x_{3}+\operatorname{sign}\left(x_{3}\right) \varepsilon_{n}\right)+\left(1-\xi\left(x_{3}\right)\right) u_{\Omega}(x) & \text { if } x \in B_{\varepsilon_{0}}^{ \pm} \backslash B_{\varepsilon_{n}}^{ \pm}, \\ u_{\Omega}(x) & \text { if } x \in \Omega_{\varepsilon_{0}},\end{cases}
$$

where $B_{\varepsilon_{0}}^{ \pm}, B_{\varepsilon_{n}}^{ \pm}$are from (2.4) and $\xi$ is a function in $\mathrm{C}_{\mathrm{c}}^{\infty}(\mathbb{R})$ such that

$$
\xi(r):= \begin{cases}1 & \text { if }|r| \leq \frac{\varepsilon_{0}}{3} \\ \in[0,1] & \text { if } \frac{\varepsilon_{0}}{3} \leq|r| \leq \frac{2 \varepsilon_{0}}{3} \\ 0 & \text { if }|r| \geq \frac{2 \varepsilon_{0}}{3}\end{cases}
$$

(namely, for $|r| \in\left[\frac{\varepsilon_{0}}{3}, \frac{2 \varepsilon_{0}}{3}\right]$ we set $\xi(r):=\xi_{r}$ with $\xi_{r}$ some element in $[0,1]$ ). Note that $\mathrm{P}_{n}^{u}\left(u_{\Omega}, u_{B}\right)$ does belong to $H_{\Gamma_{\mathrm{D}}}^{1}\left(\Omega ; \mathbb{R}^{3}\right)$ because $u=\left(u_{\Omega}, u_{B}\right)$ belongs to the space $\mathrm{U}$ from (4.11b). In what follows, we will often write $\mathrm{P}_{n}^{u}(u)$ in place of $\mathrm{P}_{n}^{u}\left(u_{\Omega}, u_{B}\right)$ for notational simplicity. We are now in a position to prove the following result.

Proposition 4.7. We have that

(1) there exists $C>0$ such that for all $\mathrm{X} \in \mathrm{H}$ there holds $\left|\mathrm{P}_{n} \mathrm{X}\right|_{n} \leq C|\mathrm{X}|$;

(2) there holds $\lim _{n \rightarrow \infty}\left|\mathrm{P}_{n} \mathrm{X}\right|_{n}=|\mathrm{X}|$,

namely properties (4.23) hold. 
Proof. We start by recalling that, for every $\mathbf{X}=(u, v) \in \mathbf{H}$ with $u=\left(u_{\Omega}, u_{B}\right)$ and $v=\left(v_{\Omega}, v_{B}\right)$ and with $\mathrm{P}_{n} \mathrm{X}=\left(\mathrm{P}_{n}^{u}(u), \mathrm{P}_{n}^{v}(v)\right)$, we have that

$$
\begin{aligned}
\left|\mathrm{P}_{n} \mathrm{X}\right|_{n}^{2}= & \int_{\Omega_{\varepsilon_{n}}} \rho^{*}\left|\mathrm{P}_{n}^{v}(v)\right|^{2} \mathrm{~d} x+\int_{B_{\varepsilon_{n}}} \rho_{n}\left|\mathrm{P}_{n}^{v}(v)\right|^{2} \mathrm{~d} x \\
& \quad+\int_{\Omega_{\varepsilon_{n}}} a e\left(\mathrm{P}_{n}^{u}(u)\right) \cdot e\left(\mathrm{P}_{n}^{u}(u)\right) \mathrm{d} x+\int_{B_{\varepsilon_{n}}} \mathrm{D} W_{\lambda_{n}, \mu_{n}}\left(e\left(\mathrm{P}_{n}^{u}(u)\right)\right) \cdot e\left(\mathrm{P}_{n}^{u}(u)\right) \mathrm{d} x \\
\doteq & I_{1}^{n}+I_{2}^{n}+I_{3}^{n}+I_{4}^{n},
\end{aligned}
$$

(cf. (4.2)), while by (4.12a) we have

$$
\begin{aligned}
|\mathrm{X}|^{2} & =\int_{\Omega} \rho^{*}\left|v_{\Omega}\right|^{2} \mathrm{~d} x+\bar{\rho} \int_{B}\left|v_{B}\right|^{2} \mathrm{~d} x+\int_{\Omega \backslash S} a e\left(u_{\Omega}\right) \cdot e\left(u_{\Omega}\right) \mathrm{d} x+\int_{B} \mathrm{D} W_{\bar{\lambda}, \bar{\mu}}\left(\partial_{3} u_{B} \otimes_{\mathrm{S}} e_{3}\right) \cdot\left(\partial_{3} u_{B} \otimes_{\mathrm{S}} e_{3}\right) \mathrm{d} x \\
& \doteq I_{1}+I_{2}+I_{3}+I_{4} .
\end{aligned}
$$

Now, by the definition (4.30) of $\mathrm{P}_{n}^{v}(v)$ we have that

$$
\begin{aligned}
I_{1}^{n}+I_{2}^{n} & =\int_{\Omega_{\varepsilon_{n}}} \rho^{*}\left|v_{\Omega}\right|^{2} \mathrm{~d} x+\int_{B_{\varepsilon_{n}}} \rho_{n}\left|\mathscr{S}_{\varepsilon_{n}}^{-1}\left[v_{B}\right]\right|^{2} \mathrm{~d} x \\
& \leq \int_{\Omega} \rho^{*}\left|v_{\Omega}\right|^{2} \mathrm{~d} x+\rho_{n} \varepsilon_{n} \int_{B}\left|v_{B}\right|^{2} \mathrm{~d} x \leq \int_{\Omega} \rho^{*}\left|v_{\Omega}\right|^{2} \mathrm{~d} x+(\bar{\rho}+c) \int_{B}\left|v_{B}\right|^{2} \mathrm{~d} x
\end{aligned}
$$

for sufficiently big $n$, where the last estimate follows from Hyp. 4.1(6). Indeed, by the dominated convergence theorem and again Hyp. 4.1 we also have

$$
I_{1}^{n}+I_{2}^{n} \rightarrow I_{1}+I_{2} .
$$

Further, taking into account that $\Omega_{\varepsilon_{n}}=\Omega_{\varepsilon_{0}} \cup\left(B_{\varepsilon_{0}}^{+} \backslash B_{\varepsilon_{n}}^{+}\right) \cup\left(B_{\varepsilon_{0}}^{-} \backslash B_{\varepsilon_{n}}^{-}\right)$and recalling the definition (4.38) of $\mathrm{P}_{n}^{u}$, we have

$$
\begin{aligned}
I_{3}^{n}=\int_{\Omega_{\varepsilon_{0}}} a e\left(u_{\Omega}\right) \cdot e\left(u_{\Omega}\right) \mathrm{d} x & +\int_{B_{\varepsilon_{0}}^{+} \backslash B_{\varepsilon_{n}}^{+}} a e\left(\xi u_{\Omega}\left(\cdot+\varepsilon_{n} e_{3}\right)+(1-\xi) u_{\Omega}\right) \cdot e\left(\xi u_{\Omega}\left(\cdot+\varepsilon_{n} e_{3}\right)+(1-\xi) u_{\Omega}\right) \mathrm{d} x \\
& +\int_{B_{\varepsilon_{0}}^{-} \backslash B_{\varepsilon_{n}}^{-}} a e\left(\xi u_{\Omega}\left(\cdot+\varepsilon_{n} e_{3}\right)-(1-\xi) u_{\Omega}\right) \cdot e\left(\xi u_{\Omega}\left(\cdot-\varepsilon_{n} e_{3}\right)+(1-\xi) u_{\Omega}\right) \mathrm{d} x
\end{aligned}
$$

and it is not difficult to check that, again by the dominated convergence theorem,

$$
I_{3}^{n} \rightarrow \int_{\Omega_{\varepsilon_{0}}} a e\left(u_{\Omega}\right) \cdot e\left(u_{\Omega}\right) \mathrm{d} x+\int_{B_{\varepsilon_{0}} \backslash S} a e\left(u_{\Omega}\right) \cdot e\left(u_{\Omega}\right) \mathrm{d} x=I_{3} .
$$

Hence, we also have that $I_{3}^{n} \leq C I_{3}$. Finally, since $\mathrm{P}_{n}^{u}(u)=u_{B}^{n}$ on $B_{\varepsilon_{n}}$, we have

$$
\begin{aligned}
I_{4}^{n} & =\int_{B_{\varepsilon_{n}}} \mathrm{D} W_{\lambda_{n}, \mu_{n}}\left(e\left(u_{B}^{n}\right)\right) \cdot e\left(u_{B}^{n}\right) \mathrm{d} x \\
& =\frac{1}{\varepsilon_{n}} \int_{B} \mathrm{D} W_{\lambda_{n}, \mu_{n}}\left(e\left(\varepsilon_{n}, u_{B, n}\right)\right) \cdot e\left(\varepsilon_{n}, u_{B, n}\right) \mathrm{d} x \\
& =\int_{B}\left(\frac{\lambda_{n}}{\varepsilon_{n}}\left|\operatorname{tr}\left(e\left(\varepsilon_{n}, u_{B, n}\right)\right)\right|^{2}+\frac{2 \mu_{n}}{\varepsilon_{n}}\left|e\left(\varepsilon_{n}, u_{B, n}\right)\right|^{2}\right) \mathrm{d} x \\
& \stackrel{(1)}{\longrightarrow} \int_{B}\left(\bar{\lambda}\left|\operatorname{tr}\left(\partial_{3} u_{B} \otimes_{\mathrm{S}} e_{3}\right)\right|^{2}+2 \bar{\mu}\left|\partial_{3} u_{B} \otimes_{\mathrm{S}} e_{3}\right|^{2}\right) \mathrm{d} x \\
& =\int_{B} \mathrm{D} W_{\bar{\lambda}, \bar{\mu}}\left(\partial_{3} u_{B} \otimes_{\mathrm{S}} e_{3}\right) \cdot\left(\partial_{3} u_{B} \otimes_{\mathrm{S}} e_{3}\right) \mathrm{d} x,
\end{aligned}
$$

where (1) is due to Hyp. $4.1(2)$ and to the strong convergence $e\left(\varepsilon_{n}, u_{B, n}\right) \rightarrow \partial_{3} u_{B} \otimes_{\mathrm{S}} e_{3}$ in $L^{2}\left(B ; \mathbb{R}_{\mathrm{sym}}^{3 \times 3}\right)$ due to Lemma 4.6(2). Clearly, these arguments also give $I_{3}^{n} \leq C I_{3}$. This concludes the proof.

Even if the convergence notion from (4.24) is the right one from the mechanical viewpoint, it could be of interest to translate this convergence in terms of some classical conventional convergence notions. 
Proposition 4.8. Let $\left(\mathrm{X}_{n}\right)_{n}=\left(u_{n}, v_{n}\right)_{n}$ with $\mathrm{X}_{n} \in \mathrm{H}_{n}$ for all $n \in \mathbb{N}$, converge in the sense of Trotter to some $\mathrm{X}=(u, v) \in \mathrm{H}$. Then, the following convergences hold as $n \rightarrow \infty$

(1) the sequence $\chi_{\Omega_{\varepsilon_{n}}}\left(u_{n}, e\left(u_{n}\right)\right)$ converge to $\left(u_{\Omega}, e\left(u_{\Omega}\right)\right)$ strongly in $L^{2}\left(\Omega ; \mathbb{R}^{3}\right) \times L^{2}\left(\Omega \backslash S ; \mathbb{R}_{\text {sym }}^{3 \times 3}\right)$;

(2) the sequence $\left.\mathscr{S}_{\varepsilon_{n}} \widehat{\left[\chi_{B_{\varepsilon_{n}}}\right.} u_{n}\right]$ converge to $\widehat{u_{B}}$ weakly in $L^{2}\left(B ; \mathbb{R}^{2}\right)$;

(3) the sequence $\left(\left(\mathscr{S}_{\varepsilon_{n}}\left[\chi_{B_{\varepsilon_{n}}} u_{n}\right]\right)_{3}, e\left(\varepsilon_{n}, \mathscr{S}_{\varepsilon_{n}}\left[\chi_{B_{\varepsilon_{n}}} u_{n}\right]\right)\right)_{n}$ converge to $\left(u_{B 3}, \partial_{3} u_{B} \otimes_{S} e_{3}\right)$ strongly in $H_{\partial_{3}}(B) \times$ $L^{2}\left(B ; \mathbb{R}_{\text {sym }}^{3 \times 3}\right) ;$

(4) moreover, if $u_{B} \in H^{1}\left(B ; \mathbb{R}^{3}\right)$, then $\mathscr{S}_{\varepsilon_{n}}\left[\chi_{B_{\varepsilon_{n}}} u_{n}\right] \rightarrow u_{B}$ strongly in $L^{2}\left(B ; \mathbb{R}^{3}\right)$;

(5) $\chi_{\Omega_{\varepsilon_{n}}} v_{n} \rightarrow v_{\Omega}$ strongly in $L^{2}\left(\Omega ; \mathbb{R}^{3}\right)$;

(6) $\mathscr{S}_{\varepsilon_{n}}\left[\chi_{B_{\varepsilon_{n}}} v_{n}\right] \rightarrow v_{B}$ strongly in $L^{2}\left(B ; \mathbb{R}^{3}\right)$.

Proof. Item (1) is an immediate consequence of the definition (4.38) of the operator $\mathrm{P}_{n}^{u}$.

As for items $(2),(3),(4)$, the key point is to observe that the convergence in the sense of Trotter of $\left(u_{n}, v_{n}\right)_{n}$ to $(u, v)$ yields that (here, for simplicity we will write $u_{n}$ in place of $\chi_{B_{\varepsilon_{n}}} u_{n}$ )

$$
\left.\left.\int_{B}\left(\frac{\lambda_{n}}{\varepsilon_{n}}\left|\operatorname{tr}\left(e\left(\varepsilon_{n}, \mathscr{S}_{\varepsilon_{n}}\left[u_{B}^{n}-u_{n}\right]\right)\right)\right|^{2}+\frac{2 \mu_{n}}{\varepsilon_{n}} \mid e\left(\varepsilon_{n}, \mathscr{S}_{\varepsilon_{n}}\left[u_{B}^{n}-u_{n}\right]\right)\right)\right|^{2}\right) \mathrm{d} x \rightarrow 0
$$

(cf. the calculations for (4.44)), whence

$$
e\left(\varepsilon_{n}, \mathscr{S}_{\varepsilon_{n}}\left[u_{B}^{n}-u_{n}\right]\right) \rightarrow 0 \text { strongly in } L^{2}\left(B ; \mathbb{R}_{\mathrm{sym}}^{3 \times 3}\right) .
$$

Combining this information with the second convergence in Lemma 4.6(2), we immediately deduce that $e\left(\varepsilon_{n}, \mathscr{S}_{\varepsilon_{n}}\left[u_{n}\right] \rightarrow \partial_{3} u_{B} \otimes_{\mathrm{S}} e_{3}\right.$ strongly in $L^{2}\left(B ; \mathbb{R}_{\text {sym }}^{3 \times 3}\right)$, whence the strong convergence of $\left(\mathscr{S}_{\varepsilon_{n}}\left[u_{n}\right]\right)_{3}$ to $u_{B 3}$. This proves item (3). Next, taking into account (4.35) we also infer that the sequence $\left(u_{B}^{n}-u_{n}\right)_{n}$ is bounded in $L^{2}\left(B ; \mathbb{R}^{3}\right)$, and then items (2) \& (4) follow from items (1) \& (3) in Lemma 4.6

As for items $(5) \&(6)$, from the Trotter convergence of $\left(u_{n}, v_{n}\right)_{n}$ to $(u, v)$ we also deduce, in particular, that

$$
\begin{array}{ll}
J_{1}^{n}:=\int_{\Omega_{\varepsilon_{n}}} \rho^{*}\left(v_{\Omega}-v_{n}\right)^{2} \mathrm{~d} x \rightarrow 0 & \text { as } n \rightarrow \infty, \\
J_{2}^{n}:=\int_{B_{\varepsilon_{n}}} \rho_{n}\left(\mathscr{S}_{\varepsilon_{n}}^{-1}\left[v_{B}\right]-\chi_{B_{\varepsilon_{n}}} v_{n}\right)^{2} \mathrm{~d} x \rightarrow 0 & \text { as } n \rightarrow \infty .
\end{array}
$$

Now, from $J_{1}^{n} \rightarrow 0$ we immediately deduce item (5); we then observe that

$$
0=\lim _{n \rightarrow \infty} J_{2}^{n}=\lim _{n \rightarrow \infty} \int_{B} \rho_{n} \varepsilon_{n}\left(v_{B}-\mathscr{S}_{\varepsilon_{n}}\left[\chi_{B_{\varepsilon_{n}}} v_{n}\right]\right)^{2} .
$$

Recalling that $\rho_{n} \varepsilon_{n} \rightarrow \bar{\rho}>0$ by Hyp. 4.1(6), we immediately infer item (6).

4.2.3. Convergence results. In order to apply Theorem 4.5 establishing the convergence in the sense of Trotter (cf. (4.24) ) of $\left(\mathrm{X}_{n}\right)_{n}$ to $\mathrm{X}$ uniformly on $[0, T]$, it is sufficient to impose suitable conditions on the initial data, which we shall discuss at the end of this section, and to check the validity of conditions (4.26) and (4.27), with the operators $\mathrm{A}_{n}: \mathrm{H}_{n} \rightrightarrows \mathrm{H}_{n}$ with domains

$$
\begin{aligned}
\mathrm{D}\left(\mathrm{A}_{n}\right):=\{\mathbf{X}=(u, v) \in \mathrm{H}:(1) & v \in H_{\Gamma_{\mathrm{D}}}^{1}\left(\Omega ; \mathbb{R}^{3}\right) \\
(2) \exists(w, \xi) \in L^{2}\left(\Omega ; \mathbb{R}^{3}\right) \times L^{2}\left(B_{\varepsilon_{n}} ; \mathbb{R}^{3}\right) \text { with } \xi \in \partial \mathcal{D}(e(v)) \text { a.e. in } B_{\varepsilon_{n}}, \text { s.t. } & \\
& \left.k_{n}\left(w, v^{\prime}\right)+\varphi_{n}\left(u, v^{\prime}\right)+b_{n} \int_{B_{\varepsilon_{n}}} \xi \cdot e\left(v^{\prime}\right) \mathrm{d} x=0 \text { for all } v^{\prime} \in H_{\Gamma_{\mathrm{D}}}^{1}\left(\Omega ; \mathbb{R}^{3}\right)\right\}
\end{aligned}
$$

defined at every $\mathrm{X}=(u, v)$ by

$$
\left.\mathrm{A}_{n} \mathbf{X}:=\left(\begin{array}{c}
-v \\
0
\end{array}\right)+\left\{\left(\begin{array}{c}
0 \\
-w
\end{array}\right): w \text { as in } 4.45 \mathrm{a}\right)(2)\right\} .
$$


and the data

$$
\left(\mathrm{F}_{n}\right)_{n},\left(\mathrm{X}_{0}^{n}\right)_{n} \quad \text { as in (3.11). }
$$

Condition (4.27) follows from the following result.

Proposition 4.9. There holds

$$
\lim _{n \rightarrow \infty}\left|\mathrm{P}_{n}\left((\mathrm{I}+\mathrm{A})^{-1}(\Psi)\right)-\left(\left(\mathrm{I}+\mathrm{A}_{n}\right)^{-1}\left(\mathrm{P}_{n}(\Psi)\right)\right)\right|_{n}=0 \quad \text { for all } \Psi \in \mathrm{H} .
$$

The proof of Proposition 4.9 is postponed after the statement of Proposition 4.11, where we are going to check (4.26). With this aim, we need to impose an additional condition on the external loading $g$.

Hypothesis 4.10. We suppose that $g \in W^{2, \infty}\left(0, T ; L^{2}\left(\Gamma_{\mathrm{N}} ; \mathbb{R}^{3}\right)\right)$ fulfills

$$
\begin{aligned}
& \operatorname{supp}(g) \cap \bar{B}_{\varepsilon_{0}}=\varnothing \quad \text { for all } t \in[0, T] \text { and } \\
& \text { if } \min \left\{\mathcal{H}^{2}\left(\Gamma_{\mathrm{D}}^{+}\right), \mathcal{H}^{2}\left(\Gamma_{\mathrm{D}}^{-}\right)\right\}=0, \text { say } \mathcal{H}^{2}\left(\Gamma_{\mathrm{D}}^{-}\right)=0, \text { then } \operatorname{supp}(g) \cap\left(\partial \Omega_{\varepsilon_{0}}^{-}\right)=\varnothing .
\end{aligned}
$$

Observe that (4.48) guarantees that the support of $g$ lies outside $\bar{B}_{\varepsilon_{0}}$ and that, if the lower adhering body is not clamped, then there are no surface forces imposed on its boundary. Under the additional Hyp. 4.10 we shall have the following result, whose proof is postponed to that of Prop. 4.9.

Proposition 4.11. There holds

(1) $\lim _{n \rightarrow \infty} \int_{0}^{T}\left|\mathrm{P}_{n}(\mathrm{~F}(t))-\mathrm{F}_{n}(t)\right|_{n} \mathrm{~d} t=0$;

(2) $\lim _{n \rightarrow \infty} \sup _{t \in[0, T]}\left|\mathrm{P}_{n}\left(\mathrm{X}^{\mathrm{e}}(t)\right)-\mathrm{X}_{n}^{\mathrm{e}}(t)\right|_{n}=0$,

where, according to the decomposition from (4.21), $\mathrm{X}^{\mathrm{e}}$ and $\mathrm{X}_{n}^{\mathrm{e}}$ are the 'stationary' parts of the solutions $\mathrm{X}$ and $\mathrm{X}_{n}$.

Let us now proceed with the proof of Proposition 4.9. which is split into three steps. The main idea is to exploit the characterizations of the resolvents of $\mathrm{A}_{n}$ and $\mathrm{A}$ provided by Proposition 3.1 and by (4.17a), respectively. In what follows, we will consider a fixed element $\Psi=\left(\psi_{1}, \psi_{2}\right) \in \mathbf{H}$.

First step: We prove the following

Lemma 4.12. For all $w \in \mathrm{U}$ there exists a sequence $\left(w_{n}\right)_{n} \subset H_{\Gamma_{\mathrm{D}}}^{1}\left(\Omega ; \mathbb{R}^{3}\right)$ such that

$$
\lim _{n \rightarrow \infty} \varphi_{n}\left(w_{n}-\mathrm{P}_{n}^{u}(w), w_{n}-\mathrm{P}_{n}^{u}(w)\right)=0
$$

and each term of

$$
\widetilde{\mathcal{J}}_{n}\left(w_{n}\right):=\frac{1}{2} \varphi_{n}\left(w_{n}, w_{n}\right)+\frac{1}{2} k_{n}\left(w_{n}, w_{n}\right)+b_{n} \int_{B_{\varepsilon_{n}}} \mathcal{D}\left(e\left(w_{n}\right)\right) \mathrm{d} x+\varphi_{n}\left(\mathrm{P}_{n}^{u}\left(\psi_{1}\right), w_{n}\right)-k_{n}\left(\mathrm{P}_{n}^{v}\left(\psi_{2}\right), w_{n}\right)
$$

converges to the corresponding term of

$$
\mathcal{J}(w):=\frac{1}{2} \varphi(w, w)+\frac{1}{2} k(w, w)+\int_{B} \overline{\mathcal{D}}\left(\partial_{3} w_{B} \otimes_{\mathrm{S}} e_{3}\right) \mathrm{d} x+\varphi\left(\psi_{1}, w\right)-k\left(\psi_{2}, w\right),
$$

cf. $4.17 \mathrm{~b}$.

Proof. Since $\mathcal{J}$ is continuous on $\mathrm{U}$, it is sufficient to prove the result on a dense subset of $\mathrm{U}$, namely the set $\left(H_{\Gamma_{\mathrm{D}}}^{1}\left(\Omega \backslash S ; \mathbb{R}^{3}\right) \times H^{1}\left(B ; \mathbb{R}^{3}\right)\right) \cap \mathrm{U}$, and to conclude via a diagonalization argument. Then, we set

$$
w_{n}:=\mathbf{P}_{n}^{u}(w) .
$$

Now, it follows from Prop. 4.7 $(2)$ that $\varphi_{n}\left(w_{n}, w_{n}\right) \rightarrow \varphi(w, w)$ as $n \rightarrow \infty$. The convergence $k_{n}\left(w_{n}, w_{n}\right) \rightarrow$ $k(w, w)$ stems from the definition (4.38) of $\mathrm{P}_{n}^{u}$ and Lemma 4.6. Indeed,

$$
k_{n}\left(w_{n}, w_{n}\right)=\int_{\Omega_{\varepsilon_{n}}} \rho^{*}\left|w_{n}\right|^{2} \mathrm{~d} x+\int_{B_{\varepsilon_{n}}} \rho_{n}\left|w_{n}\right|^{2} \mathrm{~d} x .
$$


On the one hand, again taking into account that $\Omega_{\varepsilon_{n}}=\Omega_{\varepsilon_{0}} \cup\left(B_{\varepsilon_{0}}^{+} \backslash B_{\varepsilon_{n}}^{+}\right) \cup\left(B_{\varepsilon_{0}}^{-} \backslash B_{\varepsilon_{n}}^{-}\right)$and recalling (4.38) (cf. the arguments for (4.42) $)$, we see that $\int_{\Omega_{\varepsilon_{n}}} \rho^{*}\left|w_{n}\right|^{2} \mathrm{~d} x \rightarrow \int_{\Omega} \rho^{*}\left|w_{\Omega}\right|^{2} \mathrm{~d} x$. On the other hand,

$$
\int_{B_{\varepsilon_{n}}} \rho_{n}\left|w_{n}\right|^{2} \mathrm{~d} x=\int_{B_{\varepsilon_{n}}} \rho_{n}\left|\mathscr{S}_{\varepsilon_{n}}^{-1}\left[w_{B, n}\right]\right|^{2} \mathrm{~d} x=\rho_{n} \varepsilon_{n} \int_{B}\left|w_{B, n}\right|^{2} \mathrm{~d} x \rightarrow \bar{\rho} \int_{B}\left|w_{B}\right|^{2} \mathrm{~d} x
$$

due to Hyp. 4.1(6) and Lemma 4.6(3), using that $w_{B}$ belongs to $H^{1}\left(B ; \mathbb{R}^{3}\right)$. Analogously, one can pass to the limit in the fifth contribution to $\widetilde{J}_{n}\left(w_{n}\right)$. As for the third term, we have that

$$
b_{n} \int_{B_{\varepsilon_{n}}} \mathcal{D}\left(e\left(w_{n}\right)\right) \mathrm{d} x=\frac{b_{n}}{\varepsilon_{n}^{p-1}} \int_{B} \mathcal{D}\left(e\left(\varepsilon_{n}, \mathscr{S}_{\varepsilon_{n}}\left[w_{n}\right]\right)\right) \mathrm{d} x \rightarrow \int_{B} \overline{\mathcal{D}}\left(\partial_{3} w_{B} \otimes_{\mathrm{S}} e_{3}\right) \mathrm{d} x,
$$

with $\overline{\mathcal{D}}$ given by (4.13), taking into account that $e\left(\varepsilon_{n}, \mathscr{S}_{\varepsilon_{n}}\left[w_{n}\right]\right) \rightarrow \partial_{3} w_{B} \otimes_{\mathrm{S}} e_{3}$ strongly in $L^{2}\left(B ; \mathbb{R}_{\mathrm{sym}}^{3 \times 3}\right)$ by Proposition 4.8(3). Finally, to deal with the fourth term we use that

$$
\begin{aligned}
4 \varphi_{n}\left(\mathrm{P}_{n}^{u}\left(\psi_{1}\right), \mathrm{P}_{n}^{u}(w)\right) & =\varphi_{n}\left(\mathrm{P}_{n}^{u}\left(\psi_{1}+w\right), \mathrm{P}_{n}^{u}\left(\psi_{1}+w\right)\right)-\varphi_{n}\left(\mathrm{P}_{n}^{u}\left(\psi_{1}-w\right), \mathrm{P}_{n}^{u}\left(\psi_{1}-w\right)\right) \\
& \longrightarrow \varphi\left(\psi_{1}+w, \psi_{1}+w\right)-\varphi\left(\psi_{1}-w, \psi_{1}-w\right)=4 \varphi\left(\psi_{1}, w\right)
\end{aligned}
$$

which concludes the proof.

Second step: We now show that

Lemma 4.13. Let $\bar{v}_{n}$ be the (unique) minimizer of the functional $\widetilde{\mathcal{J}}_{n}$ from (4.49) on $H_{\Gamma_{\mathrm{D}}}^{1}\left(\Omega ; \mathbb{R}^{3}\right)$. Then, there exists $\bar{v}=\left(\bar{v}_{\Omega}, \bar{v}_{B}\right) \in \mathrm{U}$ such that

(1) the elements $\widetilde{\mathrm{V}}_{n}:=\left(\bar{v}_{n}, \bar{v}_{n}\right)$ converge to $\widetilde{\mathrm{V}}:=(\bar{v}, \bar{v})$ in the sense of Trotter;

(2) every term of $\mathcal{J}(\bar{v})$ is estimated from above by the $\liminf _{n \rightarrow \infty}$ of the corresponding term of $\widetilde{\mathcal{J}}_{n}\left(\bar{v}_{n}\right)$;

(3) $\bar{v}$ is the unique minimizer of $\mathcal{J}$ on $\mathrm{U}$;

(4) $\mathcal{J}(\bar{v})=\lim _{n \rightarrow \infty} \widetilde{\mathcal{J}}_{n}\left(\bar{v}_{n}\right)$ and $|\widetilde{\mathrm{V}}|=\lim _{n \rightarrow \infty}\left|\widetilde{\mathrm{V}}_{n}\right|_{n}$.

Proof. Since $\widetilde{\mathcal{J}}_{n}\left(\bar{v}_{n}\right) \leq \widetilde{\mathcal{J}}_{n}(0)=0$, the sequence $\left(\widetilde{\mathrm{V}}_{n}\right)_{n}$ is bounded and there exists $\bar{v}=\left(\bar{v}_{\Omega}, \bar{v}_{B}\right) \in \mathrm{U}$ such that, at least along a not relabeled subsequence, there holds

(i) the sequence $\chi_{\Omega_{\varepsilon_{n}}}\left(\bar{v}_{n}, e\left(\bar{v}_{n}\right)\right)$ converges weakly to $\left(\bar{v}_{\Omega}, e\left(\bar{v}_{\Omega}\right)\right)$ in $L^{2}\left(\Omega ; \mathbb{R}^{3} \times \mathbb{R}_{\mathrm{sym}}^{3 \times 3}\right)$;

(ii) $\left(\mathscr{S}_{\varepsilon_{n}}\left[\bar{v}_{n}\right]\right)_{3}, e\left(\varepsilon_{n}, \mathscr{S}_{\varepsilon_{n}}\left[\bar{v}_{n}\right]\right)_{n}$ converges to $\left(\bar{v}_{B 3}, \partial_{3} \bar{v}_{B} \otimes_{\mathrm{s}} e_{3}\right)$ weakly in $H_{\partial_{3}}(B) \times L^{2}\left(B ; \mathbb{R}_{\mathrm{sym}}^{3 \times 3}\right)$;

(iii) $\chi_{\Omega_{\varepsilon_{n}}} \bar{v}_{n}$ converge to $\bar{v}_{\Omega}$ strongly in $L^{2}\left(\Omega ; \mathbb{R}^{3}\right)$;

(iv) $\mathscr{S}_{\varepsilon_{n}}\left[\bar{v}_{n}\right]$ converge to $\bar{v}_{B}$ weakly in $L^{2}\left(B ; \mathbb{R}^{3}\right)$.

Let us only comment on the proof of (iii): from $\int_{\Omega_{\varepsilon_{n}}}\left(\left|e\left(\bar{v}_{n}\right)\right|^{2}+\left|\bar{v}_{n}\right|^{2}\right) \mathrm{d} x \leq C$ and Korn's inequality in $\Omega_{\varepsilon_{0} / 3}$ we deduce that the sequence $\left(\bar{v}_{n}^{\text {ext }}\right)_{n}$ defined by $\bar{v}_{n}^{\text {ext }}(x):=\left(1-\xi\left(x_{3}\right)\right) \bar{v}_{n}(x)$ for all $x \in \Omega$, with $\xi$ the function from (4.39), is bounded in $H^{1}\left(\Omega ; \mathbb{R}^{3}\right)$. Hence, $\bar{v}_{n}^{\text {ext }} \rightarrow \bar{v}^{\text {ext }}$, with $\bar{v}^{\text {ext }}(x):=\left(1-\xi\left(x_{3}\right)\right) \bar{v}_{\Omega}(x)$ strongly in $L^{2}\left(\Omega ; \mathbb{R}^{3}\right)$. Likewise, Korn's inequality in $\Omega^{ \pm}$guarantees that the sequence $\left(\bar{v}_{n}^{*}\right)_{n}$ with $\bar{v}_{n}^{*}\left(\hat{x}, x_{3}\right):=\xi\left(x_{3} \pm \varepsilon_{n}\right) \bar{v}_{n}\left(\hat{x}, x_{3} \pm \varepsilon_{n}\right)$ for all $x \in \Omega^{ \pm}$is bounded in $H^{1}\left(\Omega^{ \pm} ; \mathbb{R}^{3}\right)$. Hence, $v_{n}^{*} \rightarrow v^{*}$ strongly in $L^{2}\left(\Omega ; \mathbb{R}^{3}\right)$, with $v^{*}(x):=\xi\left(x_{3}\right) \bar{v}_{\Omega}(x)$ for all $x \in \Omega$. Now, since $v_{n}^{*}$ and $\bar{v}_{n}^{\text {int }}:=\bar{v}_{n}-\bar{v}_{n}^{\text {ext }}$ weakly converge to the same limit in $L^{2}\left(\Omega ; \mathbb{R}^{3}\right)$, a fortiori we conclude that $\bar{v}_{n}=\bar{v}_{n}^{\text {ext }}+\bar{v}_{n}^{\text {int }} \rightarrow \bar{v}_{\Omega}$ in $L^{2}\left(\Omega ; \mathbb{R}^{3}\right)$.

We use the above convergences for the last two terms contributing to $\widetilde{f}_{n}\left(\bar{v}_{n}\right)$, and an additional classical lower semicontinuity argument for the first three terms to conclude Claim (2), at least along a subsequence. Then, from Step 1 we infer that $\bar{v}$ is the unique minimizer in $\mathrm{U}$ of the strictly convex functional $\mathcal{J}$, namely Claim (3). Therefore, the whole sequence $\left(\bar{v}_{n}\right)_{n}$ converge, and there holds $\mathfrak{J}(\bar{v})=\lim _{n \rightarrow \infty} \widetilde{\mathcal{J}}_{n}\left(\bar{v}_{n}\right)$. In order to 
complete the proof of Claim (4), it suffices to observe that

$$
\begin{aligned}
& \limsup _{n \rightarrow \infty}\left(\frac{1}{2} \varphi_{n}\left(\bar{v}_{n}, \bar{v}_{n}\right)+\frac{1}{2} k_{n}\left(\bar{v}_{n}, \bar{v}_{n}\right)\right) \\
& \leq \limsup _{n \rightarrow \infty}\left(\widetilde{\mathcal{J}}_{n}\left(\bar{v}_{n}\right)-b_{n} \int_{B_{\varepsilon_{n}}} \mathcal{D}\left(e\left(\bar{v}_{n}\right)\right) \mathrm{d} x-\varphi_{n}\left(\mathrm{P}_{n}^{u}\left(\psi_{1}\right), \bar{v}_{n}\right)+k_{n}\left(\mathrm{P}_{n}^{v}\left(\psi_{2}\right), \bar{v}_{n}\right)\right) \\
& =\mathcal{J}(\bar{v})-\liminf _{n \rightarrow \infty} b_{n} \int_{B_{\varepsilon_{n}}} \mathcal{D}\left(e\left(w_{n}\right)\right) \mathrm{d} x-\liminf _{n \rightarrow \infty} \varphi_{n}\left(\mathrm{P}_{n}^{u}\left(\psi_{1}\right), \bar{v}_{n}\right)+\lim _{n \rightarrow \infty} k_{n}\left(\mathrm{P}_{n}^{v}\left(\psi_{2}\right), \bar{v}_{n}\right) \\
& \leq \mathcal{J}(\bar{v})-\int_{B} \overline{\mathcal{D}}\left(\partial_{3} \bar{v}_{B} \otimes_{\mathrm{S}} e_{3}\right) \mathrm{d} x-\varphi\left(\psi_{1}, \bar{v}\right)+k\left(\psi_{2}, \bar{v}\right) \\
& =\frac{1}{2} \varphi(\bar{v}, \bar{v})+\frac{1}{2} k(\bar{v}, \bar{v}) \leq \liminf _{n \rightarrow \infty}\left(\frac{1}{2} \varphi_{n}\left(\bar{v}_{n}, \bar{v}_{n}\right)+\frac{1}{2} k_{n}\left(\bar{v}_{n}, \bar{v}_{n}\right)\right)
\end{aligned}
$$

which gives the separate convergences

$$
\varphi_{n}\left(\bar{v}_{n}, \bar{v}_{n}\right) \rightarrow \varphi(\bar{v}, \bar{v}), \quad k_{n}\left(\bar{v}_{n}, \bar{v}_{n}\right) \rightarrow k(\bar{v}, \bar{v}) .
$$

Hence, we conclude that

$$
|\widetilde{\mathrm{V}}|=\lim _{n \rightarrow \infty}\left|\widetilde{\mathrm{V}}_{n}\right|_{n}
$$

Then, we use that

$$
\left|\mathrm{P}_{n} \widetilde{\mathrm{V}}-\widetilde{\mathrm{V}}_{n}\right|_{n}^{2}=\left|\mathrm{P}_{n} \widetilde{\mathrm{V}}\right|_{n}^{2}+\left|\widetilde{\mathrm{V}}_{n}\right|_{n}^{2}-2\left(\mathrm{P}_{n} \widetilde{\mathrm{V}}, \widetilde{\mathrm{V}}_{n}\right)_{n}
$$

Taking into account (4.50) and the fact that $\lim _{n \rightarrow \infty}\left|\mathrm{P}_{n} \widetilde{\mathrm{V}}\right|_{n}^{2}=|\widetilde{\mathrm{V}}|^{2}$, in order to establish the Trotter convergence of $\left(\widetilde{\mathrm{V}}_{n}\right)_{n}$ to $\widetilde{\mathrm{V}}$ it remains to check that

$$
\lim _{n \rightarrow \infty}\left(\mathrm{P}_{n} \widetilde{\mathrm{V}}, \widetilde{\mathrm{V}}_{n}\right)_{n}=|\widetilde{\mathrm{V}}|^{2}
$$

This stems from convergences (i)-(iv). In order to check this, we use that

$$
\varphi_{n}\left(\mathrm{P}_{n}^{u}(\bar{v}), \bar{v}_{n}\right)=\int_{\Omega_{\varepsilon_{n}}} a e\left(\mathrm{P}_{n}^{u}(\bar{v})\right) \cdot e\left(\bar{v}_{n}\right) \mathrm{d} x+\int_{B_{\varepsilon_{n}}} \mathrm{D} W_{\lambda_{n}, \mu_{n}}\left(e\left(\bar{v}_{B}^{n}\right)\right) \cdot e\left(\bar{v}_{n}\right) \mathrm{d} x .
$$

To take the limit in the first term, we combine the facts that $\chi_{\Omega_{\varepsilon_{n}}} e\left(\bar{v}_{n}\right) \rightarrow e\left(\bar{v}_{\Omega}\right)$ weakly in $L^{2}\left(\Omega ; \mathbb{R}_{\mathrm{sym}}^{3 \times 3}\right)$ and $e\left(\mathrm{P}_{n}^{u}(\bar{v})\right) \rightarrow e\left(\bar{v}_{\Omega}\right)$ strongly in $L^{2}\left(\Omega \backslash S ; \mathbb{R}_{\mathrm{sym}}^{3 \times 3}\right)$ (indeed, the weak convergence of $e\left(\mathrm{P}_{n}^{u}(\bar{v})\right)$ to $e\left(\bar{v}_{\Omega}\right)$ in $L^{2}\left(\Omega \backslash S ; \mathbb{R}_{\mathrm{sym}}^{3 \times 3}\right)$ improves to a strong one by the analogue of convergence (4.43) $)$. Then, we find that

$$
\int_{\Omega_{\varepsilon_{n}}} a e\left(\mathrm{P}_{n}^{u}(\bar{v})\right) \cdot e\left(\bar{v}_{n}\right) \mathrm{d} x \rightarrow \int_{\Omega \backslash S} a e\left(\bar{v}_{\Omega}\right) \cdot e\left(\bar{v}_{\Omega}\right) \mathrm{d} x .
$$

Let us now show that

$$
\int_{B_{\varepsilon_{n}}} \mathrm{D} W_{\lambda_{n}, \mu_{n}}\left(e\left(\mathrm{P}_{n}^{u}(\bar{v})\right)\right) \cdot e\left(\bar{v}_{n}\right) \mathrm{d} x \rightarrow \int_{B} \mathrm{D} W_{\bar{\lambda}, \bar{\mu}}\left(\partial_{3} \bar{v}_{B} \otimes_{\mathrm{S}} e_{3}\right) \cdot\left(\partial_{3} \bar{v}_{B} \otimes_{\mathrm{S}} e_{3}\right) \mathrm{d} x .
$$

With this aim, we recall that $\mathrm{P}_{n}^{u}(\bar{v})=\bar{v}_{B}^{n}$ on $B_{\varepsilon_{n}}$, with $\bar{v}_{B}^{n}$ the solution to (4.31). Then, it is sufficient to observe

$$
\begin{aligned}
& \int_{B_{\varepsilon_{n}}} \mathrm{D} W_{\lambda_{n}, \mu_{n}}\left(e\left(\bar{v}_{B}^{n}\right)\right) \cdot e\left(\bar{v}_{n}\right) \mathrm{d} x \\
& =\frac{1}{\varepsilon_{n}} \int_{B} \mathrm{D} W_{\lambda_{n}, \mu_{n}}\left(e\left(\varepsilon_{n}, \mathscr{S}_{\varepsilon_{n}}\left[\bar{v}_{B}^{n}\right]\right)\right) \cdot e\left(\varepsilon_{n}, \mathscr{S}_{\varepsilon_{n}}\left[\bar{v}_{n}\right]\right) \mathrm{d} x \\
& =\frac{1}{\varepsilon_{n}} \int_{B}\left(\lambda_{n} \operatorname{tr}\left(e\left(\varepsilon_{n}, \mathscr{S}_{\varepsilon_{n}}\left[\bar{v}_{B}^{n}\right]\right)\right) \operatorname{tr}\left(e\left(\varepsilon_{n}, \mathscr{S}_{\varepsilon_{n}}\left[\bar{v}_{n}\right]\right)\right)+2 \mu_{n} e\left(\varepsilon_{n}, \mathscr{S}_{\varepsilon_{n}}\left[\bar{v}_{B}^{n}\right]\right) \cdot e\left(\varepsilon_{n}, \mathscr{S}_{\varepsilon_{n}}\left[\bar{v}_{n}\right]\right)\right) \mathrm{d} x \\
& \longrightarrow \int_{B}\left(\bar{\lambda}\left|\operatorname{tr}\left(\partial_{3} \bar{v}_{B} \otimes_{\mathrm{S}} e_{3}\right)\right|^{2}+2 \bar{\mu}\left|\partial_{3} \bar{v}_{B} \otimes_{\mathrm{S}} e_{3}\right|^{2}\right) \mathrm{d} x=\int_{B} \mathrm{D} W_{\bar{\lambda}, \bar{\mu}}\left(\partial_{3} \bar{v}_{B} \otimes_{\mathrm{S}} e_{3}\right) \cdot\left(\partial_{3} \bar{v}_{B} \otimes_{\mathrm{S}} e_{3}\right) \mathrm{d} x
\end{aligned}
$$


where the above convergence follows from the fact that $e\left(\varepsilon_{n}, \mathscr{S}_{\varepsilon_{n}}\left[\bar{v}_{B}^{n}\right]\right) \rightarrow \partial_{3} \bar{v}_{B} \otimes_{\mathrm{S}} e_{3}$ and $e\left(\varepsilon_{n}, \mathscr{S}_{\varepsilon_{n}}\left[\bar{v}_{n}\right]\right) \rightarrow$ $\partial_{3} \bar{v}_{B} \otimes_{\mathrm{S}} e_{3}$ weakly in $L^{2}\left(B ; \mathbb{R}_{\mathrm{sym}}^{3 \times 3}\right)$ by Lemmas $4.6(2)$ and convergence (ii) at the beginning of the proof of Lemma 4.13, respectively. All in all, we conclude that $\varphi_{n}\left(\mathrm{P}_{n}^{u}(\bar{v}), \bar{v}_{n}\right) \rightarrow \varphi(\bar{v}, \bar{v})$. Finally, we observe that

$$
k_{n}\left(\mathrm{P}_{n}^{v}(\bar{v}), \bar{v}_{n}\right)=\int_{\Omega_{\varepsilon_{n}}} \rho^{*} \bar{v}_{\Omega} \bar{v}_{n} \mathrm{~d} x+\int_{B_{\varepsilon_{n}}} \rho_{n} \mathscr{S}_{\varepsilon_{n}}^{-1}\left[\bar{v}_{B}\right] \bar{v}_{n} \mathrm{~d} x=\int_{\Omega} \chi_{\Omega_{\varepsilon_{n}}} \rho^{*} \bar{v}_{\Omega} \bar{v}_{n} \mathrm{~d} x+\frac{\rho_{n}}{\varepsilon_{n}} \int_{B} \bar{v}_{B} \mathscr{S}_{\varepsilon_{n}}\left[\bar{v}_{n}\right] \mathrm{d} x
$$

Then, convergences (i) and (iv) stated at the beginning of the proof yield that $k_{n}\left(\mathrm{P}_{n}^{v}(\bar{v}), \bar{v}_{n}\right) \rightarrow k(\bar{v}, \bar{v})$. We have thus established the Trotter convergence of $\left(\widetilde{\mathrm{V}}_{n}\right)_{n}$ to $\widetilde{\mathrm{V}}$.

Third step: We will show that

$$
\lim _{n \rightarrow \infty}\left|\mathrm{P}_{n} \widetilde{\mathrm{X}}-\widetilde{\mathrm{X}}_{n}\right|_{n}=0 \quad \text { with }\left\{\begin{array}{l}
\widetilde{\mathrm{X}}_{n}=\left(I+\mathrm{A}_{n}\right)^{-1}\left(\mathrm{P}_{n}(\Psi)\right) \\
\widetilde{\mathrm{X}}=(I+\mathrm{A})^{-1}(\Psi)
\end{array}\right.
$$

by exploiting the characterizations of the resolvents of $\mathrm{A}_{n}$ and $\mathrm{A}$ from (3.10) and (4.17a), respectively. Indeed, it follows from (3.10) that

$$
\widetilde{\mathrm{X}}_{n}=\left(I+\mathrm{A}_{n}\right)^{-1}\left(\mathrm{P}_{n}\left(\psi_{1}, \psi_{2}\right)\right)=\widetilde{\mathrm{V}}_{n}+\left(\mathrm{P}_{n}^{u}\left(\psi_{1}\right), 0\right),
$$

with $\widetilde{\mathrm{V}}_{n}=\left(\bar{v}, \bar{v}_{n}\right)$ the unique minimizer for the functional $\widetilde{\mathcal{J}}_{n}$ from (4.49). Analogously, one has that

$$
\widetilde{\mathrm{X}}=(I+\mathrm{A})^{-1}\left(\psi_{1}, \psi_{2}\right)=\widetilde{\mathrm{V}}+\left(\psi_{1}, 0\right)
$$

with $\widetilde{\mathrm{V}}=(\bar{v}, \bar{v})$ and $\bar{v}$ the unique minimizer of the functional $\mathcal{J}$ from (4.17b). Then,

$$
\begin{aligned}
\left|\mathrm{P}_{n} \widetilde{\mathrm{X}}-\widetilde{\mathrm{X}}_{n}\right|_{n} & =\left|\mathrm{P}_{n}\left(\widetilde{\mathrm{V}}+\left(\psi_{1}, 0\right)\right)-\widetilde{\mathrm{V}}_{n}-\left(\mathrm{P}_{n}^{u}\left(\psi_{1}\right), 0\right)\right|_{n} \\
& =\left|\mathrm{P}_{n}(\widetilde{\mathrm{V}})+\left(\mathrm{P}_{n}^{u}\left(\psi_{1}\right), 0\right)-\widetilde{\mathrm{V}}_{n}-\left(\mathrm{P}_{n}^{u}\left(\psi_{1}\right), 0\right)\right|_{n}=\left|\mathrm{P}_{n}(\widetilde{\mathrm{V}})-\widetilde{\mathrm{V}}_{n}\right|_{n} \rightarrow 0
\end{aligned}
$$

as $n \rightarrow \infty$ due to Lemma 4.13(1). Hence, (4.52) follows. This concludes the proof of Proposition 4.9

Let us now carry out the proof of Proposition 4.11 As

$$
\frac{f}{\gamma_{n}}=\chi_{\Omega_{\varepsilon_{n}}} \frac{f}{\rho^{*}}+\left(1-\chi_{\Omega_{\varepsilon_{n}}}\right) \frac{f}{\rho_{n}}
$$

and

we have that

$$
\mathrm{P}_{n}^{v}\left(\frac{f}{\rho^{*}}, 0\right)=\chi_{\Omega_{\varepsilon_{n}}} \frac{f}{\rho^{*}},
$$

$$
\left|\mathrm{P}_{n}^{v}\left(\frac{f}{\rho^{*}}, 0\right)-\left(\frac{f}{\gamma_{n}}, 0\right)\right|_{n}^{2}=\int_{B_{\varepsilon_{n}}} \frac{1}{\rho_{n}}|f|^{2} \mathrm{~d} x
$$

the first claim is a consequence of the dominated convergence theorem. As remarked in [18, condition (4.48) implies that $g \mapsto u_{n}^{\mathrm{e}}$ is a linear mapping with

$$
\varphi_{n}\left(u_{n}^{\mathrm{e}}(t), u_{n}^{\mathrm{e}}(t)\right) \leq C|g(t)|_{L^{2}\left(\Gamma_{\mathrm{N}} ; \mathbb{R}^{3}\right)} \quad \text { for all } t \in[0, T] .
$$

Since $u^{\mathrm{e}}(t)$ and $u_{n}^{\mathrm{e}}(t)$ are minimizers of $\frac{1}{2} \varphi(\cdot, \cdot)-L(t)(\cdot)$ and $\frac{1}{2} \varphi_{n}(\cdot, \cdot)-L(t)(\cdot)$, respectively, (3.1) and the arguments from the proof of Prop. 4.9 give that

$$
\lim _{n \rightarrow \infty}\left|\mathrm{P}_{n} \mathrm{X}^{\mathrm{e}}(t)-\mathrm{X}_{n}^{\mathrm{e}}(t)\right|_{n}=\lim _{n \rightarrow \infty}\left|\mathrm{P}_{n}\left(\frac{\mathrm{d} \mathrm{X}^{\mathrm{e}}}{\mathrm{d} t}(t)\right)-\frac{\mathrm{d} \mathbf{X}_{n}^{\mathrm{e}}}{\mathrm{d} t}(t)\right|_{n}=0 \quad \text { for a.a. } t \in(0, T) .
$$

But (3.1) implies that the sequence $\left(\mathrm{P}_{n} \mathrm{X}^{\mathrm{e}}-\mathrm{X}_{n}^{\mathrm{e}}\right)_{n}$ is bounded $W^{2, \infty}(0, T)$. Then, we have sufficient compactness to establish the uniform convergence in Claim (2) of Prop. 4.11. This concludes its proof.

We conclude this section by specifying the additional assumption on the initial data under which we will be able to state our convergence result. 
Hypothesis 4.14. We assume that

$$
\exists \mathrm{X}^{0} \in \mathrm{X}^{\mathrm{e}}(0)+\mathrm{D}(\mathrm{A}) ; \quad \mathrm{X}_{n}^{0} \in \mathrm{X}_{n}^{\mathrm{e}}(0)+\mathrm{D}\left(\mathrm{A}_{n}\right) \text { and } \lim _{n \rightarrow \infty}\left|\mathrm{P}_{n}\left(\mathrm{X}^{0}\right)-\mathrm{X}_{n}^{0}\right|_{n}=0 .
$$

Observe that the first condition imposes a sort of compatibility between the initial state and the initial loading conditions. The second requirement is a convergence condition that, because of Proposition 4.9, is for instance satisfied by

$$
\mathrm{X}_{n}^{0}=\mathrm{X}_{n}^{\mathrm{e}}(0)+\left(\mathrm{I}+\mathrm{A}_{n}\right)^{-1} \mathrm{P}_{n}(\mathrm{I}+\mathrm{A})^{-1}\left(\mathrm{X}^{0}-\mathrm{X}^{\mathrm{e}}(0)\right) .
$$

We are now in a position to apply the nonlinear Trotter-type Thm. 4.5 to investigate the asymptotic behavior of the solutions $\left(\mathrm{X}_{n}^{\mathrm{r}}\right)_{n} \subset W^{1, \infty}\left(0, T ; \mathrm{H}_{n}\right)$ to the Cauchy problems

$$
\left\{\begin{array}{l}
\frac{\mathrm{d}}{\mathrm{d} t} \mathrm{X}(t)+\mathrm{A}_{n} \mathrm{X}(t) \ni \mathrm{F}_{n}(t) \\
\mathbf{X}(0)=\mathrm{X}_{0}^{n}-\mathrm{X}^{\mathrm{e}}(0),
\end{array} \quad \text { in } \mathrm{H}_{n} \text { for a.a. } t \in(0, T),\right.
$$

in the Hilbert spaces $\left(\mathrm{H}_{n},|\cdot|_{n}\right)$ from (4.2), with the operators $\left(\mathrm{A}_{n}\right)_{n}$ from (4.45) and the data $\mathrm{F}_{n}$ from (4.46). Therefore, we deduce that the sequence $\left(\mathrm{X}_{n}^{\mathrm{r}}\right)_{n}$ converges uniformly, in the sense of Trotter, to the solution $\mathrm{X}^{\mathrm{r}}$ to the Cauchy problem (4.19), with the initial datum $X_{0}^{r}=X_{0}-X^{e}(0)$. This is summarized in the following theorem, which is the main result of the paper.

Theorem 4.15. Assume Hypotheses 4.1, 4.2, 4.10, and 4.14. Then, the solutions $\left(\mathrm{X}_{n}\right)_{n}$ to the Cauchy problems

$$
\left\{\begin{array}{l}
\frac{\mathrm{d}}{\mathrm{d} t} \mathrm{X}_{n}+\mathrm{A}_{n}\left(\mathrm{X}_{n}-\mathrm{X}_{n}^{\mathrm{e}}\right) \ni\left(0, \frac{f}{\gamma_{n}}\right) \quad \text { a.e. in }(0, T), \\
\mathrm{X}_{n}(0)=\mathrm{X}_{n}^{0}
\end{array}\right.
$$

converge to the solution $\mathbf{X}$ of the Cauchy problem

$$
\left\{\begin{array}{l}
\frac{\mathrm{d}}{\mathrm{d} t} \mathrm{X}+\mathrm{A}\left(\mathrm{X}-\mathrm{X}^{\mathrm{e}}\right) \ni(0, f / \bar{\rho}) \quad \text { a.e. in }(0, T), \\
\mathrm{X}(0)=\mathrm{X}^{0}
\end{array}\right.
$$

in the sense that

$$
\lim _{n \rightarrow \infty}\left|\mathrm{P}_{n}(\mathbf{X}(t))-\mathrm{X}_{n}(t)\right|_{n}=0 \quad \text { uniformly in }[0, T]
$$

and, in addition, there holds

$$
\lim _{n \rightarrow \infty}\left|\mathbf{X}_{n}(t)\right|_{n}=|\mathbf{X}(t)| \quad \text { uniformly in }[0, T] .
$$

While convergence (4.57) is guaranteed by Thm. 4.5, cf. (4.28), the additional (4.58) follows from observing that the sequence $\left(\left|\mathrm{X}_{n}(\cdot)\right|_{n}-|\mathrm{X}(\cdot)|\right)_{n}$ is bounded in $W^{1, \infty}(0, T)$, and therefore equicontinuous. This turns the pointwise convergence to 0 into uniform convergence on $[0, T]$.

Finally, let us highlight that, in view of Proposition 4.11(2), from Thm. 4.15 we also infer the uniform convergence of the sequence $\mathrm{X}_{n}=\mathrm{X}_{n}^{\mathrm{r}}+\mathrm{X}_{n}^{\mathrm{e}}$.

\section{Conclusive Results AND REMARKS}

The variational formulation corresponding to (4.56). In this final section we gain further insight into the variational formulation of the initial-boundary value problem encompassed in the Cauchy problem (4.56). We will distinguish the cases $\bar{b}<\infty$ and $\bar{b}=\infty$. 
When $\underline{\bar{b}}$ is finite, a more explicit way of writing (4.56) is

$\exists \xi \in \partial \overline{\mathcal{D}}\left(\partial_{3} v_{B} \otimes_{\mathrm{S}} e_{3}\right)$ such that

$$
\begin{aligned}
& \int_{\Omega} \rho^{*} \partial_{t t} u_{\Omega} \cdot \psi_{\Omega} \mathrm{d} x+\int_{\Omega \backslash S} a e\left(u_{\Omega}\right) \cdot e\left(\psi_{\Omega}\right) \mathrm{d} x+\int_{B} \bar{\rho} \partial_{t t} u_{B} \cdot \psi_{B} \mathrm{~d} x+\int_{B}\left(\mathrm{D} W_{\bar{\lambda}, \bar{\mu}}\left(\partial_{3} u_{B} \otimes_{\mathrm{S}} e_{3}\right)+\xi\right) \cdot\left(\partial_{3} \psi_{B} \otimes_{\mathrm{S}} e_{3}\right) \mathrm{d} x \\
& =\int_{\Omega} f \cdot \psi_{\Omega} \mathrm{d} x+\int_{\Gamma_{\mathrm{N}}} g \cdot \psi_{\Omega} \mathrm{d} \mathcal{H}^{2}(x) \\
& \quad \text { for all } \psi \in\left\{\left(\psi_{\Omega}, \psi_{B}\right) \in H_{\Gamma_{\mathrm{D}}}^{1}\left(\Omega \backslash S ; \mathbb{R}^{3}\right) \times H_{\partial_{3}}\left(B ; \mathbb{R}^{3}\right): \gamma_{S^{ \pm}}\left(\psi_{B}\right)=\gamma_{S}\left(\psi_{\Omega}^{ \pm}\right)\right\}, \quad \text { in }(0, T),
\end{aligned}
$$

supplemented with suitable initial conditions. Hence, the limiting behavior may be described in terms of a coupled system of two evolutionary, or transient, problems set in $\Omega \backslash S$ and in $B$. Clearly, the stress and the displacement fields $\sigma_{\Omega}$ and $u_{\Omega}$ in the limiting adhering bodies that occupy $\Omega^{+}$and $\Omega^{-}$satisfy the following relations, written in strong form

$$
\begin{aligned}
& \rho^{*} \partial_{t t} u_{\Omega}-\operatorname{div} \sigma_{\Omega}=f \\
& \sigma_{\Omega}=a e\left(u_{\Omega}\right) \\
& u_{\Omega}=0 \\
& \sigma_{\Omega} n=g \\
& \mp\left(\sigma_{\Omega}^{ \pm} e_{3}\right)(\hat{x}, t)=\frac{1}{2} \int_{-1}^{1}\left[\bar{\rho}\left(1 \pm x_{3}\right) \partial_{t t} u_{B}\left(\hat{x}, x_{3}, t\right)\right. \\
& \left. \pm\left(\left(\left(\mathrm{D} W_{\bar{\lambda}, \bar{\mu}}\left(\partial_{3} u_{B}\left(\hat{x}, x_{3}, t\right) \otimes_{\mathrm{S}} e_{3}\right)+\xi\right) e_{3}\right) \cdot e_{3}\right) e_{3}\right] \mathrm{d} x_{3} \quad \text { on } S \times(0, T) .
\end{aligned}
$$

This corresponds to the transient response to the loading $(f, g)$ of each adhering body clamped on $\Gamma_{\mathrm{D}}^{ \pm}$and linked through a mechanical constraint along $S$. Differently from the case of an adhesive layer with a vanishing total mass, which was considered in [18, the contact between the bodies need not be described only in terms of the traces $\gamma_{S}\left(u_{\Omega}^{ \pm}\right), \gamma_{S}\left(v_{\Omega}^{ \pm}\right)$of the displacement and velocity of the sole adhering bodies. In fact, one has to consider the additional variables $\left(u_{B}, v_{B}=\partial_{t} u_{B}\right)$ which keep track of the dynamics of the adhesive layer. These variables fulfill the following equations

$$
\begin{array}{ll}
\bar{\rho} \partial_{t t} u_{B}-\partial_{3}\left(\sigma_{B} e_{3}\right)=0 & \text { in } B \times(0, T), \\
\sigma_{B} \in \mathrm{D} W_{\bar{\lambda}, \bar{\mu}}\left(\partial_{3} u_{B} \otimes_{\mathrm{S}} e_{3}\right)+\partial \overline{\mathcal{D}}\left(\partial_{3} u_{B} \otimes_{\mathrm{S}} e_{3}\right) & \text { in } B \times(0, T), \\
\gamma_{S^{ \pm}}\left(u_{B}\right)=\gamma_{S}\left(u_{\Omega}^{ \pm}\right) & \text {on } S \times(0, T) .
\end{array}
$$

Systems (5.2) and (5.3) are supplemented by suitable initial conditions. Such equations are of the same type as those in the original layer. Of course, the variables $\left(u_{B}, v_{B}\right)$ may be eliminated and, consequently (see (5.2) ), the contact condition along $S$ between the two adhering bodies is a nonlocal - in time, only- relation between the stress vector $\left.\sigma_{\Omega}^{ \pm}(\hat{x}, t)\right) e_{3}$ at the current time $t$, and the history of $\gamma_{S}\left(u_{\Omega}^{ \pm}(\hat{x}, \tau)\right)$, with $\tau \in[0, t]$. Finally, from the last line of (5.2) we deduce that

$$
\left(\sigma_{\Omega}^{-} e_{3}\right)(\hat{x}, t)-\left(\sigma_{\Omega}^{+} e_{3}\right)(\hat{x}, t)=\int_{-1}^{1} \bar{\rho} \partial_{t t} u_{B}\left(\hat{x}, x_{3}, t\right) \mathrm{d} x_{3}
$$

which reflects the fact that the jump of the stress vector on the adhering bodies balances the limiting inertial forces stemming from the adhesive.

In the case $\underline{\bar{b}}=\infty$, the system reads

$$
\begin{aligned}
& \partial_{3} v_{B}=0 \text { and } \\
& \int_{\Omega} \rho^{*} \partial_{t t} u_{\Omega} \psi \mathrm{d} x+\int_{\Omega \backslash S} a e\left(u_{\Omega}\right) \cdot e(\psi) \mathrm{d} x+2 \bar{\rho} \int_{S} \partial_{t} v_{\Omega} \psi \mathrm{d} x=\int_{\Omega} f \cdot \psi \mathrm{d} x+\int_{\Gamma_{\mathrm{N}}} g \cdot \psi \mathrm{d} \mathcal{H}^{2}(x)
\end{aligned}
$$


for all $\psi \in H_{\Gamma_{\mathrm{D}}}^{1}\left(\Omega ; \mathbb{R}^{3}\right)$, in $(0, T)$, again supplemented by suitable initial conditions. Indeed, from $\partial_{3} v_{B}=0$, supposing that the initial datum for $u_{B}$ is independent of the variable $x_{3}$ we deduce that $\partial_{3} u_{B}=0$, and hence that $\llbracket u_{\Omega} \rrbracket=0$. Hence the space for the test functions in (5.4) is $H_{\Gamma_{\mathrm{D}}}^{1}\left(\Omega ; \mathbb{R}^{3}\right)$. Let us stress that if $\bar{b}=\infty$ then the relative motion along $S$ is frozen.

Other relative behavior of the parameters $\left(\lambda_{n}, \mu_{n}\right)$. As previously mentioned, the analysis in Section 4 has been carried out confining the discussion to the case in which the parameters $(\bar{\lambda}, \bar{\mu})$ are in $[0, \infty) \times(0, \infty)$. Let us conclude the paper by examining the singular cases

(1) $(\bar{\lambda}, \bar{\mu}) \in\{\infty\} \times(0, \infty)$

(2) $(\bar{\lambda}, \bar{\mu}) \in[0, \infty) \times\{\infty\}$;

(3) $(\bar{\lambda}, \bar{\mu}) \in(0, \infty) \times\{0\}$;

(4) $\bar{\lambda}=\bar{\mu}=0$.

In each of these cases, we will explicitly illustrate spaces $\mathrm{H}, \mathrm{U}, \mathrm{V}$, the bilinear forms $\varphi$ and $k$, the operator $\mathrm{A}$, and the function $u^{\mathrm{e}}$, like we have done for the case $(\bar{\lambda}, \bar{\mu}) \in[0, \infty) \times(0, \infty)$. We will not give the proof of the convergence result, as it is a straightforward adaptation of that developed throughout Sec. 4.2, and we will leave it to the interested reader. Nonetheless, we will hint at the main point underlying the identification of the limit problem, namely the correct identification of the space $\mathrm{U}$ which, in turn, will be based on the analysis of the asymptotic behavior of a sequence $\left(u_{n}\right)_{n}$ with $\sup _{n} \varphi_{n}\left(u_{n}, u_{n}\right)<\infty$, cf. the arguments in Section 4.1 .

Let us start by specifying that in each of the above cases we will have

$$
\begin{aligned}
& \mathrm{H}=\mathrm{U} \times \mathrm{V}, \\
& \mathrm{V}=L^{2}\left(\Omega ; \mathbb{R}^{3}\right) \times L^{2}\left(B ; \mathbb{R}^{3}\right), \\
& \left(\mathrm{X}, \mathrm{X}^{\prime}\right)=\varphi\left(u, u^{\prime}\right)+k\left(v, v^{\prime}\right) \text { for all } \mathrm{X}=(u, v), \mathbf{X}^{\prime}=\left(u^{\prime}, v^{\prime}\right) \in \mathrm{H},
\end{aligned}
$$

with the bilinear form $k$ given by (4.12C), the form $\varphi$ specified in (5.6b), (5.7b), (5.8b), and (5.9b) below, the space $\mathrm{U}$ specified in (5.6a), (5.7a), (5.8a), (5.9a) below, the operator $\mathrm{A}: \mathrm{H} \rightrightarrows \mathrm{H}$ with domain è

$$
\begin{aligned}
& \mathrm{D}(\mathrm{A})=\{\mathrm{X} \in \mathrm{H}:(\mathrm{i}) v \in \mathcal{P}, \\
& \text { (ii) } \exists(w, \xi) \in \mathrm{V} \times \partial \overline{\mathcal{D}}\left(\partial_{3} v_{B} \otimes_{\mathrm{S}} e_{3}\right) \text { s.t. } \\
& \left.k(w, \psi)+\varphi(u, \psi)+\int_{B} \xi \cdot\left(\partial_{3} \psi_{B} \otimes_{\mathrm{S}} e_{3}\right) \mathrm{d} x=0 \quad \text { for all } \psi \in \overline{\mathrm{U}}\right\},
\end{aligned}
$$

and defined by

$$
\mathrm{AX}:=\left(\begin{array}{c}
-v \\
0
\end{array}\right)+\left\{\left(\begin{array}{c}
0 \\
-w
\end{array}\right): w \text { as in (5.5a) }(2)\right\} .
$$

The space $\bar{U}$ featuring in (5.5a) (2) will be specified for each of the singular cases considered (cf. (5.6c), (5.7c), (5.8c), (5.9c) below); analogously, $\tilde{v}$ shall be built from $v \in \mathcal{P}$ in a way depending on the case under consideration (cf. (5.6d), (5.7d), (5.8d), (5.9d) below), with the space

$$
\mathcal{P}=\left\{\psi=\left(\psi_{\Omega}, \psi_{B}\right) \in H^{1}\left(\Omega \backslash S ; \mathbb{R}^{3}\right) \times H_{\partial_{3}}\left(B ; \mathbb{R}^{3}\right): \gamma_{S^{ \pm}}\left(\psi_{B}\right)=\gamma_{S}\left(\psi_{\Omega}^{ \pm}\right)\right\} .
$$

Case 1: $(\bar{\lambda}, \bar{\mu}) \in\{\infty\} \times(0, \infty)$. In this case we have (recall the notation $\left.\llbracket z \rrbracket=\gamma_{S}\left(z^{+}\right)-\gamma_{S}\left(z^{-}\right)\right)$

$$
\begin{aligned}
& \mathrm{U}=\left\{u=\left(u_{\Omega}, u_{B}\right) \in H_{\Gamma_{\mathrm{D}}}^{1}\left(\Omega \backslash S ; \mathbb{R}^{3}\right) \times H_{\partial_{3}}\left(B ; \mathbb{R}^{3}\right): \llbracket u_{\Omega} \rrbracket_{3}=0, \partial_{3} u_{B 3}=0\right\}, \\
& \varphi\left(u, u^{\prime}\right)=\int_{\Omega \backslash S} a e\left(u_{\Omega}\right) \cdot e\left(u_{\Omega}^{\prime}\right) \mathrm{d} x+\bar{\mu} \int_{B} \partial_{3} \widehat{u}_{B} \cdot \partial_{3} \widehat{u}_{B}^{\prime} \mathrm{d} x, \\
& \overline{\mathrm{U}}=\mathrm{U}, \\
& \tilde{v}=v .
\end{aligned}
$$


Indeed, the condition $\frac{\lambda_{n}}{\varepsilon_{n}} \rightarrow \infty$ implies that, along a sequence $\left(u_{n}\right)_{n}$ with $\sup _{n} \varphi_{n}\left(u_{n}, u_{n}\right)<\infty$, there holds $\operatorname{tr}\left(e\left(\varepsilon_{n}, \mathscr{S}_{\varepsilon_{n}}\left[u_{n}\right]\right)\right) \rightarrow 0$ in $L^{2}(B)$, whence the condition $\partial_{3} u_{B 3}=0$ and, consequently, $\llbracket u_{\Omega} \rrbracket_{3}=0$, as encompassed in the space $U$ from (5.6a). Here, the adhering bodies are in bilateral contact along $S$ and the tangential component of the stress vector applied along $S$ is given by

$$
\gamma_{S} \widehat{\left(\sigma_{\Omega}^{ \pm}\right)} e_{3}= \pm \frac{1}{2} \int_{-1}^{1}\left(\bar{\rho}\left(1 \pm x_{3}\right) \partial_{t t} \widehat{u}_{B} \pm\left(\bar{\mu} \partial_{3} \widehat{u}_{B}+\widehat{\xi e_{3}}\right)\right) \mathrm{d} x_{3} \quad \text { on } S \times(0, T)
$$

with

$$
\begin{cases}\xi \in \partial \overline{\mathcal{D}}\left(\partial_{3} v_{B} \otimes_{S} e_{3}\right) & \text { in } B \times(0, T), \\ \bar{\rho} \partial_{t t} \widehat{u}_{B}-2 \bar{\mu} \frac{\partial^{2} \widehat{u}_{B}}{\frac{\partial x_{3}^{2}}{2}-\frac{\partial}{\partial x_{3}} \widehat{\xi e_{3}}=0} & \text { in } B \times(0, T), \\ \left.\widehat{\gamma_{S^{ \pm}}\left(u_{B}\right.}\right)=\gamma_{S}\left(u_{\Omega}^{ \pm}\right) & \text {on } S \times(0, T),\end{cases}
$$

supplemented by suitable initial conditions. Observe that only the tangential component of the traces on $S$ of the displacement in the adhering bodies is nonlocal-in-time. This is a kind of viscoelastic behavior with long memory when $\bar{b}$ is finite. When $\bar{b}=\infty$, since $\partial_{3} v_{B}=0$, the relative sliding along $S$ is frozen.

Case $2:(\bar{\lambda}, \bar{\mu}) \in[0, \infty) \times\{\infty\}$. We have

$$
\begin{aligned}
& \mathrm{U}=H_{\Gamma_{\mathrm{D}}}^{1}\left(\Omega ; \mathbb{R}^{3}\right), \\
& \varphi\left(u, u^{\prime}\right)=\int_{\Omega \backslash S} a e\left(u_{\Omega}\right) \cdot e\left(u_{\Omega}^{\prime}\right) \mathrm{d} x, \\
& \overline{\mathrm{U}}=\left\{v \in \mathcal{P}: \partial_{3} v_{B}=0, \llbracket v_{\Omega} \rrbracket=0\right\}, \\
& \tilde{v}=v_{\Omega} .
\end{aligned}
$$

In this case, the condition $\frac{\mu_{n}}{\varepsilon_{n}} \rightarrow \infty$ implies that $e\left(\varepsilon_{n}, \mathscr{S}_{\varepsilon_{n}}\left[u_{n}\right]\right) \rightarrow 0$ in $L^{2}\left(B ; \mathbb{R}_{\mathrm{sym}}^{3 \times 3}\right)$, so that $\partial_{3} u_{B}=0$ and, consequently, $\llbracket u_{\Omega} \rrbracket=0$. Hence $u_{\Omega}$ satisfies

$$
\int_{\Omega} \rho^{*} \partial_{t t} u_{\Omega} \cdot \psi \mathrm{d} x+\int_{S} 2 \bar{\rho} \partial_{t t} u_{\Omega} \cdot \psi \mathrm{d} \hat{x}+\int_{\Omega} a e\left(u_{\Omega}\right) \cdot e(\psi) \mathrm{d} x=\int_{\Omega} f \cdot \psi \mathrm{d} x+\int_{\Gamma_{\mathrm{N}}} g \cdot \psi \mathrm{d} \mathcal{H}^{2}(x)
$$

for all $\psi \in H_{\Gamma_{\mathrm{D}}}^{1}\left(\Omega ; \mathbb{R}^{3}\right)$, in $(0, T)$. The adhering bodies are perfectly bonded and the obtained deformable body is submitted to surface forces on $S$ corresponding to the limit of the vertical forces in the adhesive.

Case 3: $(\bar{\lambda}, \bar{\mu}) \in(0, \infty) \times\{0\}$. Here we find

$$
\begin{aligned}
& \mathrm{U}=\left\{u=\left(u_{\Omega}, u_{B 3}\right) \in H_{\Gamma_{\mathrm{D}}}^{1}\left(\Omega \backslash S ; \mathbb{R}^{3}\right) \times H_{\partial_{3}}(B):\left(\gamma_{S^{ \pm}}\left(u_{B}\right)\right)_{3}=\left(\gamma_{S}\left(u_{\Omega}^{ \pm}\right)\right)_{3}\right\}, \\
& \varphi\left(u, u^{\prime}\right)=\int_{\Omega \backslash S} a e\left(u_{\Omega}\right) \cdot e\left(u_{\Omega}^{\prime}\right) \mathrm{d} x+\bar{\lambda} \int_{B} \partial_{3} u_{B 3} \cdot \partial_{3} u_{B 3}^{\prime} \mathrm{d} x, \\
& \overline{\mathrm{U}}=\mathcal{P}, \\
& \tilde{v}=\left(v_{\Omega}, v_{B 3}\right) .
\end{aligned}
$$

In this case, from $\varphi_{n}\left(u_{n}, u_{n}\right) \leq C$ we may only infer the information that $\left(\operatorname{tr}\left(e\left(\varepsilon_{n}, \mathscr{S}_{\varepsilon_{n}}\left[u_{n}\right]\right)\right)\right)_{n}$ is bounded in $L^{2}(B)$, and thus only $u_{B 3}$ is defined and belongs to $H_{\partial_{3}}(B)$, with the condition $\left(\gamma_{S^{ \pm}}\left(u_{B}\right)\right)_{3}=\left(\gamma_{S}\left(u_{\Omega}^{ \pm}\right)\right)_{3}$. Every adhering body is subjected to surface forces along $S$ given by

$$
\gamma_{S}\left(\sigma_{\Omega}^{ \pm}\right) e_{3}= \pm \frac{1}{2} \int_{-1}^{1}\left(\bar{\rho}\left(1 \pm x_{3}\right) \partial_{t}\left(\widehat{v}_{B}, \partial_{t} u_{B 3}\right) \pm\left(\bar{\lambda} \partial_{3} u_{B 3} e_{3}+\xi e_{3}\right)\right) \mathrm{d} x_{3} \quad \text { on } S \times(0, T)
$$

with

$$
\begin{cases}\bar{\rho} \partial_{t}\left(\widehat{v}_{B}, \partial_{t} u_{B 3}\right)-\frac{\partial}{\partial x_{3}}\left(\sigma_{B} e_{3}\right)=0 & \text { in } B \times(0, T), \\ \sigma_{B} \in \bar{\lambda} \partial_{3} u_{B 3} e_{3} \otimes_{\mathrm{S}} e_{3}+\partial \overline{\mathcal{D}}\left(\partial_{3} v_{B} \otimes_{S} e_{3}\right) & \text { in } B \times(0, T), \\ \left.\left(\gamma_{S^{ \pm}}\left(u_{B}\right)\right)_{3}=\left(\gamma_{S}\left(u_{\Omega}^{ \pm}\right)\right)_{3}, \quad \widehat{\gamma_{S^{ \pm}}\left(v_{B}\right.}\right)=\widehat{\gamma_{S}\left(u_{\Omega}^{ \pm}\right)} & \text {on } S \times(0, T),\end{cases}
$$

supplemented by suitable initial conditions. In this case, the forces are a nonlocal-in-time function (of the traces on $S$ of the displacements of both adhering bodies) of viscoelastic with long-memory type. 
Case $4: \bar{\lambda}=\bar{\mu}=0$. Here

$$
\begin{aligned}
& \mathrm{U}=\left\{u=u_{\Omega} \in H_{\Gamma_{\mathrm{D}}}^{1}\left(\Omega \backslash S ; \mathbb{R}^{3}\right)\right\}, \\
& \varphi\left(u, u^{\prime}\right)=\int_{\Omega \backslash S} a e\left(u_{\Omega}\right) \cdot e\left(u_{\Omega}^{\prime}\right) \mathrm{d} x, \\
& \overline{\mathrm{U}}=\mathcal{P}, \\
& \tilde{v}=v_{\Omega} .
\end{aligned}
$$

Here, the estimate $\varphi_{n}\left(u_{n}, u_{n}\right) \leq C$ yields no information. That is why, the only conditions are given on $v_{\Omega}$ and $v_{B}$; they are involved in the domain of the operator A (cf. (5.5a)) and specified by the space $\overline{\mathrm{U}}$ from (5.9c). Each adhering body is subject to surface forces along $S$ given by

$$
\gamma_{S}\left(\sigma_{\Omega}^{ \pm}\right) e_{3}= \pm \frac{1}{2} \int_{-1}^{1}\left(\bar{\rho}\left(1 \pm x_{3}\right) \partial_{t} v_{B} \pm \xi e_{3}\right) \mathrm{d} x_{3} \quad \text { on } S \times(0, T),
$$

with

$$
\begin{cases}\bar{\rho} \partial_{t} v_{B}-\frac{\partial}{\partial x_{3}}\left(\xi e_{3}\right)=0 & \text { in } B \times(0, T), \\ \xi \in \partial \overline{\mathcal{D}}\left(\partial_{3} v_{B} \otimes_{\mathrm{S}} e_{3}\right) & \text { in } B \times(0, T), \\ \gamma_{S^{ \pm}}\left(v_{B}\right)=\gamma_{S}\left(v_{\Omega}^{ \pm}\right) & \text {on } S \times(0, T),\end{cases}
$$

supplemented by a suitable initial condition. These forces are a nonlocal-in-time function (of the traces on $S$ of the velocity in both adhering bodies) of viscous with long-memory type.

\section{REFERENCES}

[1] Bonetti E., Bonfanti G., Lebon F., Rizzoni R., A model of imperfect interface with damage, Meccanica, 52 (8), 1911-1922, (2017).

[2] Bonetti E., Bonfanti G., Lebon F., Derivation of imperfect interface models coupling damage and temperature, Computers and Mathematics with Applications, 11, 2906-2916, (2019).

[3] Bonetti E., Bonfanti G., Rossi R., Global existence for a contact problem with adhesion, Math. Meth. Appl. Sci., 31, 1029-1064, (2008).

[4] Bonetti E., Bonfanti G., Rossi R., Thermal effects in adhesive contact: modelling and analysis, Nonlinearity, 22, 2967-2731, (2009).

[5] Bonetti E., Bonfanti G., Rossi R., Modeling via internal energy balance and analysis of adhesive contact with friction in thermoviscoelasticity, Nonlin. Anal. RWA, 22, 473-507, (2015).

[6] H. Brezis, Opérateurs Maximaux-Monotones et Semi-Groupes de Contraction dans les Espaces de Hilbert, North-Holland, 1972.

[7] Dumont S., Lebon, F., Rizzoni, R., An asymptotic approach to the adhesion of thin stiff films, Mech. Res. Com., 58, 24-35, (2014).

[8] Freddi L., Paroni, R., Roubǐček T., Zanini C., Quasistatic delamination models for Kirchhoff-Love plates, ZAMM Z. Angew. Math. Mech., 91, 845-865, (2011).

[9] Freddi L., Roubiček T., Zanini C., Quasistatic delamination of sandwich-like Kirchhoff-Love plates, J. Elast., 113, 219-250, (2013).

[10] Frémond M., Adhérence des solides, J. Mec. Theor. Appl., 6, 383-407, (1987).

[11] Frémond M., Non-Smooth Thermo-mechanics, Springer (2001).

[12] Lebon, F., Rizzoni, R., Asymptotic behavior of a hard thin linear elastic interphase: An energy approach, Int. J. Sol. Struct., 48, 441-449, (2011).

[13] Licht C., Weller T., Approximation of semi-groups in the sense of Trotter and asymptotic mathematical modeling in physics of continuous media, Discrete Contin. Dyn. Syst. Ser. S, 12, 1709-1741, (2019).

[14] Klarbring A., Derivation of the adhesively bonded joints by the asymptotic expansion method, Int. J. Eng. Sci., 29, 493-512, (1991).

[15] Kočvara M., Mielke A., Roubíček T., A rate-independent approach to the delamination problem, Math. Mech. Solids, 11, $423-447,(2006)$.

[16] Licht C., Comportement asymptotique d'une bande dissipative mince de faible rigidité, C. R. Acad. Sci. Paris Sér. I Math., 317, 429-433, (1993).

[17] Licht C., Michaille G., A modelling of elastic bonded joints, Adv. Math. Sci. Appl., 7, 711-740, (1997). 
[18] C. Licht, A. Léger, S. Orankitjaroen, and A. Ould Khaoua: Dynamics of elastic bodies connected by a thin soft viscoelastic layer. J. Math. Pures Appl., 99, 685-703, (2013).

[19] Mielke A., Roubíček T., Thomas M., From damage to delamination in nonlinear elastic materials at small strains, J. Elast., 109, 235-273, (2012).

[20] O. Iosifescu, C. Licht, and G. Michaille, Nonlinear boundary conditions in Kirchhoff-Love plate theory, J. Elast., 96, 57-79, (2009).

[21] Roubíček T., Scardia L., Zanini C., Quasistatic delamination problem, Contin. Mech. Thermodyn., 21, 223-235, (2009).

[22] Trotter, H. F. Approximation of semi-groups of operators. Pacific J. Math., 8, 887-919, (1958).

E. Bonetti, Dipartimento di Matematica, Università degli studi di Milano, via Saldini 50, 20133 Milano - Italy

E-mail address: elena.bonetti @ unipv.it

G. Bonfanti, DiCATAM - Sezione di Matematica, Università degli studi di Brescia, Via Valotti 9 , 25133 Brescia ITALY

E-mail address: giovanna.bonfanti @unibs.it

C. Licht, LMGC, Univ Montpellier, CnRS, Montpellier, France and Dept. Maths, Mahidol University, Bangkok, Thailand

E-mail address: christian.licht@ umontpellier.fr

R. Rossi, Dimi, Università degli studi di Brescia, via Branze 38, 25133 Brescia - Italy

E-mail address: riccarda.rossi @ unibs.it 